\title{
Kinetic theory for electron transmission through a molecular wire
}

\author{
E.G. Petrov ${ }^{a, *}$, V. May ${ }^{b}$, P. Hänggi ${ }^{c}$ \\ ${ }^{a}$ Bogolyubov Institute for Theoretical Physics, National Academy of Sciences of Ukraine, Metrologichna str., 14-b, UA-03143 Kiev, Ukraine \\ ${ }^{\mathrm{b}}$ Institut für Physik, Humboldt Universität zu Berlin, Newtonstraße 15, D-12489 Berlin, Germany \\ ' Institut für Physik, Universität Augsburg, D-86135 Augsburg, Germany
}

Received 9 December 2004; accepted 24 March 2005

Available online 23 May 2005

\begin{abstract}
A theoretical description of electron transmission through a molecular wire embedded in between two leads is carried out using the density matrix method. Accounting for the Coulomb repulsion among the transferred electrons nonlinear kinetic equations for the reduced single-electron distributions are derived. The respective transfer rates contain contributions from different transmission channels which are characterized by the number of excess electrons present in the wire in the course of the charge transmission. Special attention is focused on the study of single-electron transmission. It is shown that a direct lead-lead (elastic) transmission as well as a transmission including the population of intermediate wire states (inelastic transmission) becomes possible if the electron to be transferred moves through a wire without a further excess charge. The probability to find a molecule in such an "empty" wire state follows from a relation between the rates of incoming and outgoing lead-molecule/molecule-lead charge transfer. In turn, they are responsible for the formation of the inelastic component of the current. Thus, it could be demonstrated that the inelastic charge transmission not only determines the inelastic part of the current but is able to control the elastic component as well. Moreover, the inelastic transmission may result in a specific kinetic rectification effect.
\end{abstract}

(C) 2005 Elsevier B.V. All rights reserved.

PACS: $05.20 . \mathrm{Dd} ; 05.60 .+\mathrm{w}$

Keywords: Molecule wire; Interelectrode current; Kinetic equations

\section{Introduction}

Molecular wires represent one example of nanostructures where the diversity of molecular structures may influence the electron transfer in a characteristic way [1-11]. Such a pronounced structural control of charge transfer and thus of the molecular wire-mediated current, additionally, can also be achieved by the application of external dc- and ac-fields [12-16]. (A discussion of the various methods to control distant electron transfer through molecular systems can be found in [3-5,17-19] and the reviews $[8,20,21]$.) The description of the wire-mediated inter-electrode tunnel process may be undertaken for the case where all units of a linear molecule form the bridging structure for electron transmission (elastic inter-electrode tunneling, see [3-5,12], or tunneling including energy dissipation at the bridged sites, cf. $[19,22])$. Alternatively, one may consider the case where the terminal sites of the wire localize the transferred electron (inelastic inter-electrode tunneling mediated by the terminal sites, see [6,23-26]). Any of these studies underlined the

\footnotetext{
* Corresponding author.

E-mail addresses: epetrov@bitp.kiev.ua (E.G. Petrov), may@physik.hu-berlin.de (V. May), hanggi@physik.uni-augsburg.de (P. Hänggi).
} 
importance of relaxation processes disturbing the charge transmission. Relaxation processes are mainly caused by the interaction of electronic degrees of freedom with environmental vibrations. Moreover, intramolecular vibrational modes are involved in electron transmission, too, forming phonon sidebands [27]. Recent theoretical studies on the phonon-assisted inelastic tunnel current and the related conductance gave good insight into the particular nature of the vibronic states related to single-molecule electron transport (see, e.g., [28-30] and especially [31]). It has been also shown that the reorganization energy associated with the nuclear displacements and caused by the electron transmission through the molecule plays a key role in controlling charge localization as well as inelastic scattering events [25]. Additionally, the importance of the Coulomb interaction among different transferred electrons has been underlined in $[5,9,32-36]$.

It is typical for the huge variety of molecular metals, conducting polymers, and supramolecular compounds fabricated so far (see, e.g., [37-39]) that the charge transmission within them is dominated by relaxation processes (leading to the destruction of coherency). Electron motion in macromolecular systems like oligoporphyrin and polythiophene structures [7], electrically conductive metallomacrocyclic assemblies [40], molecular chains containing transition metal complexes [41], metal containing fullerene structures [42] and others [39,43,44] is mainly hopping transport between the different sites of electron localization. In contrast, nonlinearities in the current-voltage $(I-V)$ characteristics of single molecules (see [45-57]) have been explained in the Landauer-Büttiker theory [58-60], which exclusively assumes elastic scattering processes of the electron moving through the molecular wire [55,56,60-62]. However, inelastic processes should also influence the current through the molecular wire [25,26,47,63-65]. A respective unified theory should describe elastic and inelastic processes, probably, also accounting for the Coulomb interaction among different transferred electrons (or holes).

The goal of the present paper is to undertake such a unified description. We will consider electron transmission through a molecule/molecular wire characterized by molecular orbitals (MOs) extending over the whole molecule. If embedded between two electrodes the system "left lead-molecule-right lead" (LMR) is formed. Charge transmission through the LMR system which is accompanied by a population of the MOs cause the inelastic component of the current. However, the same MOs act as scattering centers in a direct (elastic) lead-lead transmission what results in the elastic component of inter-electrode current. Both types of charge transmission will be described in the framework of the well-established density matrix theory [66-69]. A similar approach has been already used for a unified description of bridge mediated electron transfer in donor-acceptor complexes [70,71]. To account for many-electron effects in the course of single-electron transmission the occupation number representation will be utilized and the derivation of nonlinear kinetic equations is demonstrated.

The paper is organized as follows. Section 2 introduces the model used to describe electron transfer in the LMR system. Nonlinear kinetic equations governing electron transport to a molecular wire including inter-electron Coulomb repulsion are derived in Section 3. The corresponding transfer rates characterizing single-electron transitions between multi-electron states of the LMR system are also presented. Section 4 concentrates on single-electron transmission. A comparison between the direct lead-lead electron transmission and the transmission through intermediate states of the LMR system is given in Section 5. Moreover, the conditions are identified at which the overall current through a singleelectron transmission channel is given by additive contributions from elastic and the inelastic transfer processes. The Conclusions offer a general discussion on the importance of inelastic processes on formation of the inter-electrode current.

\section{Model and basic Hamiltonian}

Let a linear molecule (molecular wire) be embedded in between two leads (cf. Fig. 1). If an excess electron leaves a lead and enters the molecule it can be localized within one of $N$ molecular sites. We will employ a simple tightbinding model where each molecular site is characterized by a single MO $|m\rangle$. The energy of the transferred extra electron at the $m$ th molecular site, $E_{m}(Q)$ (here, $Q$ denotes the set of vibrational coordinates leading to a modulation of the electronic energy), and transfer coupling between neighboring sites, $V_{m m \pm 1}$, define the electronic part of the molecular Hamiltonian. Introducing electron creation and annihilation operators $a_{m \sigma}^{+}$and $a_{m \sigma}$, respectively, we may write

$$
H_{M}^{(\mathrm{el})}=\sum_{m, m^{\prime}} \sum_{\sigma}\left[E_{m}(Q) \delta_{m, m^{\prime}}+V_{m m^{\prime}}\left(\delta_{m^{\prime}, m+1}+\delta_{m^{\prime}, m-1}\right)\right] a_{m \sigma}^{+} a_{m^{\prime} \sigma} .
$$

The summation covers all sites $\left(m, m^{\prime}=1,2, \ldots, N\right)$ and all electronic spin projections $(\sigma= \pm 1 / 2)$. To get the complete molecular Hamiltonian $H_{M}$ one has to add a kinetic energy of nuclei. 


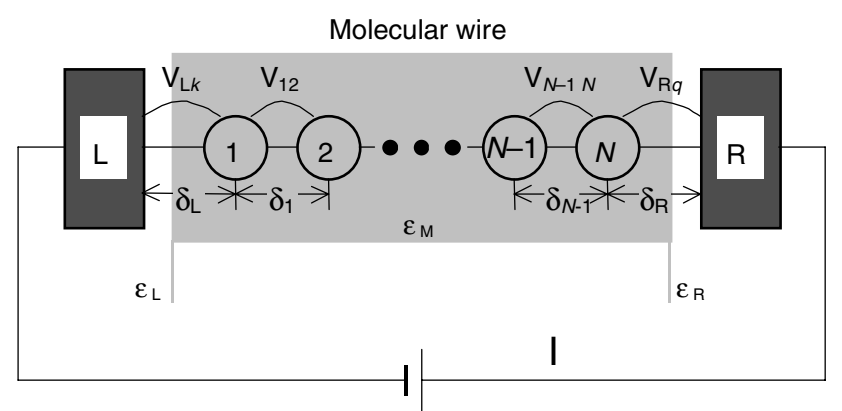

Fig. 1. The molecular wire ( $N$-site linear molecule) embedded in between two leads. The inter-site matrix elements $V_{12}, V_{13}, \ldots, V_{N-1 N}$ lead to the formation of the extended MOs of the molecule. The coupling to the leads is covered by the $V_{L \mathbf{k}}$ and $V_{R \mathbf{q}}$. The quantities $\delta_{L}=l_{L} / \epsilon_{L}, \delta_{R}=l_{R} / \epsilon_{R}$, and $\delta_{m}=l_{m} / \epsilon_{M}$ denote the effective lengths ( $l_{L}$ and $l_{R}$ are the distances between the terminal molecular sites and respective lead surfaces, $l_{m}$ is the distance between neighboring molecular sites). The quantities $\epsilon_{L}\left(\epsilon_{R}\right)$ and $\epsilon_{M}$ denote the permitivities near the left (right) lead and in the vicinity of the molecule, respectively. The effective distance between the leads is $\delta=\delta_{L}+\sum_{m=1}^{N-1} \delta_{m}$.

Below we will consider the case of strong intersite transfer coupling. Therefore, extended MOs, defined via

$$
|\mu\rangle=\sum_{m} u_{\mu}(m)|m\rangle,
$$

have to be introduced. The $u_{\mu}(m)$ are found via a diagonalization of Hamiltonian $H_{M}^{(\text {el })}$, Eq. (1) at $E_{m}=E_{m}\left(Q_{0}\right)$ where $Q_{0}$ denotes the set of equilibrium nuclear coordinates. The related energies are denoted as $\varepsilon_{\mu}$. Now we use a conventional scheme $[69,72]$. To derive an electron-vibrational coupling one may expand the $H_{M}$ with respect to the displacements $Q-Q_{0}$. Introducing the vibrational eigenstates $\left\langle\chi_{\mu \alpha_{\mu}}\right\rangle$ for each $\mu$ th $\mathrm{MO}$ one obtains (for more details see, e.g., [73])

$$
H_{M}=H_{M}^{(0)}+V_{M-M}+V_{M-B} .
$$

The first part,

$$
H_{M}^{(0)}=\sum_{\mu, \alpha_{\mu}} \sum_{\sigma} \varepsilon_{\mu \alpha_{\mu}} a_{\mu \sigma}^{+} a_{\mu \sigma}\left|\chi_{\mu \alpha_{\mu}}\right\rangle\left\langle\chi_{\mu \alpha_{\mu}}\right|,
$$

defines the electron-vibrational states, where $\varepsilon_{\mu \alpha_{\mu}}$ and $a_{\mu \sigma}^{+}\left(a_{\mu \sigma}\right)$ denote the electron-vibrational eigenenergy and the electron creation (annihilation) operator, respectively. (The latter are connected with the $a_{m \sigma}^{+}\left(a_{m \sigma}\right)$ via the transformation (2).) The coupling expression

$$
V_{M-M}=\sum_{\mu, \mu^{\prime}} \sum_{\alpha_{\mu}, \beta_{\mu^{\prime}}} \sum_{\sigma}\left(1-\delta_{\mu, \mu^{\prime}}\right) V_{\mu \alpha_{\mu} \mu^{\prime} \beta_{\mu^{\prime}}} a_{\mu \sigma}^{+} a_{\mu^{\prime} \sigma}\left|\chi_{\mu \alpha_{\mu}}\right\rangle\left\langle\chi_{\mu^{\prime} \beta_{\mu^{\prime}}}\right|
$$

is responsible for transitions among different electron-vibrational states, whereas

$$
V_{M-B}=\sum_{\mu \sigma} \sum_{\alpha_{\mu}, \alpha_{\mu}^{\prime}}\left(1-\delta_{\alpha_{\mu}, \alpha_{\mu}^{\prime}}\right) \Phi_{\alpha_{\mu} \alpha_{\mu}^{\prime}}^{(\mu)} a_{\mu \sigma}^{+} a_{\mu \sigma}\left|\chi_{\mu \alpha_{\mu}}\right\rangle\left\langle\chi_{\mu \alpha_{\mu}^{\prime}}\right|
$$

describes transitions between different vibrational levels of the same electronic state. Note, that matrix elements $V_{\mu \alpha_{\mu} \mu^{\prime} \beta_{\mu^{\prime}}}$ and $\Phi_{\alpha_{\mu} \alpha_{\mu}^{\prime}}^{(\mu)}$ depend on additional vibrational degrees of freedom forming a thermal bath, thus the Hamiltonians (5) and (6) allow to describe relaxation processes within the molecule.

Embedding the considered molecule in between two leads, we have to account for the additional lead-molecule coupling. Just the details of this coupling are of crucial importance for the electron transmission through the molecule $[10,47,74,75]$. We will consider the leads as a macroscopic system with a continuous spectrum associated with the conduction band. The Hamiltonian covering the states of the two leads can be written as

$$
H_{L R}=\sum_{r=L, R} \sum_{\mathbf{k}, \sigma} E_{r \mathbf{k}} a_{r \mathbf{k} \sigma}^{+} a_{r \mathbf{k} \sigma}
$$

where $E_{r \mathbf{k}}$ is the energy of an electron with wave vector $\mathbf{k}$ in the conduction band of the $r$ th lead. To specify the leadmolecule interaction we introduce the vibrational energy and the $\lambda$ th vibrational state of the molecule in the absence of any excess electrons as $\varepsilon_{\lambda}^{(0)}$, and $\left|\chi_{\lambda}\right\rangle$, respectively. Moreover, $V_{L \mathbf{k}}\left(V_{R \mathbf{k}}\right)$ is the transfer coupling between the first $(N \mathrm{th})$ molecular site and the left (right) lead. The lead-molecule interaction follows as

$$
V_{L R-M}=\sum_{\mu \sigma} \sum_{\lambda \alpha_{\mu}} \sum_{r=L, R}\left[V_{r \mathbf{k} \lambda, \mu \alpha_{\mu}} a_{r \mathbf{k} \sigma}^{+} a_{\mu \sigma}\left|\chi_{\lambda}\right\rangle\left\langle\chi_{\mu \alpha_{\mu}}\right|+\text { h.c. }\right] \text {. }
$$


Here, the lead-molecule coupling matrix elements

$$
V_{L \mathbf{k} \lambda, \mu \alpha_{\mu}}=V_{L \mathbf{k}} u_{\mu}(1)\left\langle\chi_{\lambda} \mid \chi_{\mu \alpha_{\mu}}\right\rangle, \quad V_{R \mathbf{k} \lambda, \mu \alpha_{\mu}}=V_{R \mathbf{k}} u_{\mu}(N)\left\langle\chi_{\lambda} \mid \chi_{\mu \alpha_{\mu}}\right\rangle
$$

account for the MOs extending over the whole molecule (via $u_{\mu}(1)$ and $u_{\mu}(N)$ ) and include the vibrational overlap integrals $\left\langle\chi_{\lambda} \mid \chi_{\mu \alpha_{\mu}}\right\rangle$. In most cases the molecule-lead coupling is realized via specific terminal groups which are bonded to the leads either directly or through sulfur [54,61,62] or silicon [76] atoms. In any case, the empty levels of terminal groups should be energetically positioned far above the LUMOs of the molecule so that the molecule-lead couplings $V_{L \mathbf{k}}$ and $V_{R \mathbf{k}}$, Eq. (9) are originated by superexchange mechanisms between the surface atoms of the lead and the terminal groups of the molecule. Accordingly, charge transmission through the molecule is related to the terminal sites $m=1$ and $m=N$ as well as to the sites $m=2,3, \ldots, N-1$. The corresponding single-electron energies $\varepsilon_{\mu \alpha_{\mu}}$, Eq. (4) correspond to the extended states formed by the localized states of all $N$ sites of the molecule.

All terms discussed so far are comprised in the LMR-system Hamiltonian

$$
H=H_{L R}+H_{M}+H_{L R-M}+H_{\text {Coul }} \text {. }
$$

All following derivations of kinetic equations and of the interelectrode current will be based on it. The Hamiltonian contains contributions formed by single-electron states as well as the coupling of these states to vibrational substates (see Eqs. (3)-(8)). Moreover, we included $H_{\text {Coul }}$ referring to the Coulomb repulsion between different excess electrons. It has to be considered whenever more then a single excess electron participates in transmission through a molecule.

\section{Nonlinear kinetic equations}

The current formation through the molecular wire is characterized by coherent charge motion as well as relaxation processes. We will assume that only intra-molecular relaxation modifies the coherent electron transmission. These processes are governed by the Hamiltonian $V_{M-B}$, Eq. (6) and proceed on a picosecond time scale [72,77]. To compare this time scale with the time $\tau_{\operatorname{tr}}$ of charge motion through the molecule let us use the relation $I \sim|e| / \tau_{\operatorname{tr}}$ with $I$ being the current and $|e|$ the absolute value of the electron charge. Measured values of the current through single molecules may reach 1 up to $100 \mathrm{nA}$, leading to $\tau_{\mathrm{tr}}$ in the order of $10^{-10}-10^{-12} \mathrm{~s}$. Therefore, the intramolecular current proceeds against the background of fast vibrational relaxation, and a coarse-grained description of the electron motion becomes possible. Accordingly, the current can be expressed via integral electronic state populations $P_{j \sigma_{j}}(t)$. Here, the index $j$ indicates the electronic lead states $(j=L \mathbf{k}, R \mathbf{q})$ and the electronic levels of the molecule $(j=f)$, whereas $\sigma_{j}$ denotes the electron spin.

The stationary current $I$ may be obtained from the general relation

$$
I=e \dot{N}_{L}
$$

where $\dot{N}_{L}\left(=-\dot{N}_{R}\right)$ is the time-derivative of the electron number of the left (right) electrode. Since the total left electrode population is determined as

$$
N_{L}(t)=\sum_{\mathbf{k} \sigma_{\mathbf{k}}} P_{L \mathbf{k} \sigma_{\mathbf{k}}}(t)
$$

we have to determine the time-derivative of the single electron state distribution $P_{L \mathbf{k} \sigma_{\mathbf{k}}}(t)$ (here, $\sigma_{\mathbf{k}}$ denotes spin which belongs to the electron with wave vector $\mathbf{k}$ ). To calculate the total current we have to derive kinetic equations for the distribution functions $P_{R \mathbf{q} \sigma_{\mathbf{q}}}(t)$ as well as for the populations $P_{f \sigma_{f}}(t)$ of the electronic levels of the molecule.

In the problem under consideration, more then one excess electrons can occupy the molecule in the course of electron transmission. Therefore, a Coulomb interaction between the transferred electrons modifies the single-electron transmission. To account for this fact in kinetic equations for single electron distribution functions $P_{j \sigma_{j}}(t)(j=L \mathbf{k}$, $R \mathbf{q}, f)$, one has to derive kinetic equations for multi-electron distribution functions (populations) $\mathscr{P}_{\{N\}}(t)$ where $\{N\}=\left\{N_{L \mathbf{k} \sigma_{\mathbf{k}}}\right\}\left\{N_{R \mathbf{q} \sigma_{q}}\right\}\left\{N_{f \sigma_{f}}\right\}$ denotes the complete set of LMR electronic occupation numbers. Here, $\left\{N_{L \mathbf{k} \sigma_{\mathbf{k}}}\right\}$ and $\left\{N_{R \mathbf{q} \sigma_{\mathbf{q}}}\right\}$ are the sets related respectively to the left and right lead occupation numbers whereas $\left\{N_{f \sigma_{f}}\right\}$ refers to the molecular occupation numbers. If kinetic equation for the $\mathscr{P}_{\{N\}}(t)$ is known then using the definitions

$$
P\left(N_{j \sigma_{j}}, t\right)=\sum_{\{N\} \neq N_{j \sigma_{j}}} \mathscr{P}_{\{N\}}(t)
$$

and

$$
P_{j \sigma_{j}}(t)=\sum_{N_{j \sigma_{j}}=0,1} N_{j \sigma_{j}} P\left(N_{j \sigma_{j}}, t\right)
$$


as well as normalization conditions

$$
\sum_{N_{j \sigma_{j}}=0,1} P\left(N_{j \sigma_{j}}, t\right)=1
$$

one comes to kinetic equations for desirable overall single-electron populations (distribution functions) $P_{j \sigma_{j}}(t)$. [Note that in line with relation (14) populations $P_{j \sigma_{j}}(t)$ and $1-P_{j \sigma_{j}}(t)$ are directly connected with the occupation number distribution functions $P\left(N_{j \sigma_{j}}, t\right)$, with respectively $N_{j \sigma_{j}}=1$ at the presence of an electron in the $j$ th state with spin-projection $\sigma_{j}$ and with $N_{j \sigma_{j}}=0$ at its absence.] Each $P\left(N_{j \sigma_{j}}, t\right)$ follows by a summation of the multi-electron population $\mathscr{P}_{\{N\}}(t)$ with respect to all occupation numbers except $N_{j \sigma_{j}}$.

\subsection{Single-electron and multi-electron LMR states}

In the present paper, the specification of the single-electron states $\left|j \sigma_{j}\right\rangle$ is achieved in the framework of a model where the leads are considered as macroscopic systems. It has been shown in Appendix A, that in such a case the influence of the lead-molecule interaction on the lead states $\left|L(R) \mathbf{k} \sigma_{\mathbf{k}}\right\rangle$ as well as the lead energy levels $E_{L(R) \mathbf{k}}$ may be ignored. At the same time, this interaction strongly influences the electron-vibrational states $\left|\mu \alpha_{\mu}\right\rangle \equiv\left|1_{\mu \sigma_{\mu}}\right\rangle\left|\chi_{\mu \alpha_{\mu}}\right\rangle=$ $a_{\mu \sigma_{\mu}}^{+}\left|0_{\mu \sigma_{\mu}}\right\rangle\left|\chi_{\mu \alpha_{\mu}}\right\rangle$ as well as the electron-vibrational energy levels $\varepsilon_{\mu \alpha_{\mu}}$ of the molecule. In line with the results obtained in the Appendix, the molecular electron-vibrational states $\left|f \alpha_{f}\right\rangle=\sum_{\mu \alpha_{\mu}} \Theta_{f \alpha_{f}}\left(\mu \alpha_{\mu}\right)\left|\mu \alpha_{\mu}\right\rangle$ and the corresponding singleelectron energies $\mathscr{E}_{f \alpha_{f}}$ are derived from a set of algebraic equations (A.42) which cover an effective molecular Hamiltonian

$$
H_{M}^{\text {(eff) }}=\sum_{\mu \alpha_{\mu}} \sum_{\mu^{\prime} \alpha_{\mu^{\prime}}}\left[E_{\mu \alpha_{\mu}} \delta_{\mu \alpha_{\mu}, \mu^{\prime} \alpha_{\mu^{\prime}}}+\sum_{r=L, R} \sum_{\mu \alpha_{\mu} \mu^{\prime} \alpha_{\mu^{\prime}}}^{(r)}\right]\left|\mu \alpha_{\mu}\right\rangle\left\langle\mu^{\prime} \alpha_{\mu^{\prime}}\right| .
$$

Hamiltonian (16) takes into account the broadening of molecular levels caused by vibrational relaxation (via the energies $E_{\mu \alpha_{\mu}}$, Eq. (A.9)) as well as by the influence of the macroscopic leads (via the self-energies $\Sigma_{\mu \alpha_{\mu} \mu^{\prime} \alpha_{\mu^{\prime}}}^{(r)}$ Eq. (A.40)). When diagonalized it obeys the form

$$
H_{M}^{(\text {eff })}=\sum_{f} \sum_{\sigma_{f} \alpha_{f}} \mathscr{E}_{f \alpha_{f}} a_{f \sigma}^{+} a_{f \sigma}\left|\chi_{f \alpha_{f}}\right\rangle\left\langle\chi_{f \alpha_{f}}\right| .
$$

[Note that $\left.\left|f \alpha_{f}\right\rangle\left\langle f \alpha_{f}\left|=a_{f \sigma_{f}}^{+} a_{f \sigma_{f}}\right| \chi_{f \alpha_{f}}\right\rangle\left\langle\chi_{f \alpha_{f}}\right|.\right]$ If off-diagonal parts of self-energies $\Sigma_{\mu \alpha_{\mu} \mu^{\prime} \alpha_{\mu^{\prime}}}^{(r)}$ are of less importance for a coupling between different molecular states (2), i.e. if the inequality

$$
\left|\mathscr{E}_{\mu \alpha_{\mu}}-\mathscr{E}_{\mu^{\prime} \alpha_{\mu^{\prime}}}\right| \gg\left|\sum_{\mu \alpha_{\mu} \mu^{\prime} \alpha_{\mu^{\prime}}}^{(L)}\right|,\left|\sum_{\mu \alpha \mu \mu^{\prime} \alpha_{\mu^{\prime}}}^{(R)}\right|
$$

is satisfied in the precise LMR system, the proper energy $\mathscr{E}_{f \alpha_{f}}$ takes a simple form

$$
\mathscr{E}_{f \alpha_{f}} \approx \mathscr{E}_{\mu \alpha_{\mu}}=\epsilon_{\mu \alpha_{\mu}}-\mathrm{i} \Gamma_{\mu \alpha_{\mu}} .
$$

Here, the quantity $\epsilon_{\mu \alpha_{\mu}}=\varepsilon_{\mu \alpha_{\mu}}+\sum_{r=L, R} \operatorname{Re} \sum_{\mu \alpha_{\mu} \mu \alpha_{\mu}}^{(r)}$ is the renormalized energy of the electron-vibrational state $\left|\mu \alpha_{\mu}\right\rangle$ of the $\mu$ th extended single-electron molecular level, and

$$
\Gamma_{\mu \alpha_{\mu}}=\gamma_{\mu \alpha_{\mu}}+\sum_{r=L, R} \Gamma_{\mu \alpha_{\mu}}^{(r)}
$$

defines the integral broadening of the $\mu \alpha_{\mu}$ th electron-vibrational energy level. The broadening contains a contribution $\gamma_{\mu \alpha_{\mu}}$ originated by relaxation processes in the given molecular level as well as the contributions $\Gamma_{\mu \alpha_{\mu}}^{(r)}=\operatorname{Im} \Sigma_{\mu \alpha_{\mu} \mu \alpha_{\mu}}^{(r)}$ which result from the molecule-lead interaction.

Once single-electron states $\left|L \mathbf{k} \sigma_{\mathbf{k}}\right\rangle,\left|R \mathbf{q} \sigma_{\mathbf{k}}\right\rangle,\left|f \alpha_{f}\right\rangle$ and respective single-electron energies $E_{L \mathbf{k}}, E_{R \mathbf{q}}, \mathscr{E}_{f \alpha_{f}}$ have been specified, the problem arises to construct the multi-electron states and the multi-electron energies. Generally, multielectron states have to include the information on molecular spin which is able to change its value in the course of electron transmission through a molecule (see, for instance $[12,21,23,64])$. But, if the exchange interaction associated with the transferred electrons gives a minor contribution in energy of the LMR system one can specify the spin states of multi-electron system only fixing the spin projections of separate electrons (the leads are assumed to be fabricated from nonmagnetic metals). It is definitely happen when a molecule does not contain the paramagnetic ions and, additionally, the location of the transferred electrons within the molecule occurs at well separated sites. It is not the case when the excess electrons occupy the strongly delocalized MOs. As such, the exchange interaction between the transferred (excess) electrons can be ignored only, if special physical conditions are satisfied. 
As an example, we consider a Coulomb interaction written in the Hubbard form [78]

$$
H_{\text {Coul }}=U \sum_{m} a_{m \uparrow}^{+} a_{m \uparrow} a_{m \downarrow}^{+} a_{m \downarrow} .
$$

The parameter $U$ characterizes the repulsion between two transferred electrons occupying the same localized MO at the $m$ th molecular site but having different spin projections, $+1 / 2 \equiv \uparrow$ and $-1 / 2 \equiv \downarrow$. In the basis of extended MOs, Eq. (2) $H_{\text {Coul }}$ changes to

$$
\begin{aligned}
& H_{\text {Coul }}=U \sum_{\mu_{1}, \mu_{1}^{\prime}} \sum_{\mu_{2}, \mu_{2}^{\prime}} \Phi\left(\mu_{1} \mu_{1}^{\prime} ; \mu_{2} \mu_{2}^{\prime}\right) a_{\mu_{1} \uparrow}^{+} a_{\mu_{1}^{\prime}} a_{\mu_{2} \downarrow}^{+} a_{\mu_{2}^{\prime} \downarrow} \\
& \left(\Phi\left(\mu_{1} \mu_{1}^{\prime} ; \mu_{2} \mu_{2}^{\prime}\right)=\Phi\left(\mu_{2} \mu_{2}^{\prime} ; \mu_{1} \mu_{1}^{\prime}\right)=\sum_{m} u_{\mu_{1}}^{*}(m) u_{\mu_{1}^{\prime}}(m) u_{\mu_{2}}^{*}(m) u_{\mu_{2}^{\prime}}(m)\right) .
\end{aligned}
$$

The Hamiltonian includes single-electron as well as two-electron transitions between the extended molecular states. Note that the two-electron transitions reflects the direct as well as the exchange scattering processes. But, if the inequality

$$
\left|\left(\epsilon_{\mu_{1}}+\epsilon_{\mu_{2}}+U \Phi\left(\mu_{1} \mu_{1} ; \mu_{2} \mu_{2}\right)\right)-\left(\epsilon_{\mu_{1}^{\prime}}+\epsilon_{\mu_{2}^{\prime}}+U \Phi\left(\mu_{1}^{\prime} \mu_{1}^{\prime} ; \mu_{2}^{\prime} \mu_{2}^{\prime}\right)\right)\right| \gg U\left|\Phi\left(\mu_{1} \mu_{1}^{\prime} ; \mu_{2} \mu_{2}^{\prime}\right)\right|\left(1-\delta_{\mu_{1}, \mu_{1}^{\prime}}\right)\left(1-\delta_{\mu_{2}, \mu_{2}^{\prime}}\right)
$$

is satisfied for any set of extended states $\mu$, then the single-electron transitions mainly contributes to the transition processes. Following from this the terms with $\mu_{1}=\mu_{1}^{\prime}$ or $\mu_{2}=\mu_{2}^{\prime}$ should dominate. If the additional inequality

$$
\left|\tilde{\epsilon}_{\mu}-\tilde{\epsilon}_{\mu^{\prime}}\right| \gg U \sum_{v}\left|\Phi\left(\mu \mu^{\prime} ; v v\right)\right| \quad\left(\tilde{\epsilon}_{\mu} \equiv \epsilon_{\mu}+U \sum_{v} \Phi(\mu \mu ; v v)\right)
$$

is valid then the electron-electron interaction can be described in using the truncated form of the Hamiltonian $H_{\text {Coul. }}$. It reads

$$
H_{\mathrm{Coul}} \approx H_{\mathrm{Coul}}^{\text {(trunc) }}=U \sum_{\mu, v} \Phi(\mu \mu ; v v) a_{\mu \uparrow}^{+} a_{\mu \uparrow} a_{v \downarrow}^{+} a_{v \downarrow} .
$$

Accordingly, the use of the Hamiltonian from Eq. (25) instead that of Eq. (22) is justified either for a large energy gap $\left|\epsilon_{\mu}-\epsilon_{\mu^{\prime}}\right|$ between single-electron extended states or due to a large energy difference between local molecular levels (as compared with site-site electronic couplings $V_{m m^{\prime}}$, Eq. (1)). Moreover we note that the Hamiltonian, Eq. (25) is diagonal with respect to the occupation numbers and thus does not contain the exchange interaction between the excess electrons occupying the extended MOs. Matrix elements of the Hamiltonian (25) are diagonal and read

$$
U\{N\}=U \sum_{\mu, v} \Phi(\mu \mu ; v v) N_{\mu \uparrow} N_{v \downarrow} .
$$

In the present description we restrict ourself to the case where exchange interaction associated with the transferred electrons is of less importance and thus one can apply a Hartree approach. The Hartree approach indicates that a total LMR electronic state $|a\rangle \equiv\left|\{N\}, v\left(\left\{N_{f \sigma_{f}}\right\}\right)\right\rangle$ appears as a product of single-electron states,

$$
|a\rangle=\prod_{\mathbf{k} \sigma_{\mathbf{k}}}\left|N_{L \mathbf{k} \sigma_{\mathbf{k}}}\right\rangle \prod_{\mathbf{q} \sigma_{\mathbf{q}}}\left|N_{R \mathbf{q} \sigma_{\mathbf{q}}}\right\rangle \prod_{f \sigma_{f}}\left|N_{f \sigma_{f}}\right\rangle\left|v\left(\left\{N_{f \sigma_{f}}\right\}\right)\right\rangle .
$$

It means that a LMR spin state is determined by a set of electronic spin-projections. Multi-electron states (27) contain the set of molecular vibrational states denoted through the $\left|v\left(\left\{N_{f \sigma_{f}}\right\}\right)\right\rangle$. Within the set each separate vibronic state coincides either with the $\left|\chi_{f \alpha_{f}}\right\rangle$ (extra electron occupies the $f$ th MO, $N_{f \sigma_{f}}=1$ ) or with $\left|\chi_{\lambda}\right\rangle$ (no extra electron at the $f$ th MO, $\left.\sum_{\sigma_{f}} N_{f \sigma_{f}}=0\right)$. The states (27) are the proper states of the effective LMR Hamiltonian

$$
H_{L M R}^{(\text {eff })}=H_{L R}+H_{M}^{(\text {eff })}+H_{\text {Coul }}
$$

where the terms in the right side of Eq. (28) are defined by Hamiltonians (7), (17) and (25), respectively.

\subsection{Kinetic equations for the multi-electron distribution function}

The kinetic equations for the $\mathscr{P}_{\{N\}}(t)$ are obtained from a generalized master equation (GME) which is obeyed by the reduced density operator $\rho(t)$ describing the dynamics of an open quantum system. The main problem here is to simultaneously account for the fast relaxational transitions among the vibrational substates of each molecular level, the lead-molecule coupling, and the Coulomb interaction between the excess electrons. To offer a sufficient clear description we separately consider in Appendix A the derivation of a master equation for the single-electron 
distribution functions. It is demonstrated how the fast vibrational relaxation as well as the lead-molecule interaction justifies a reduced description via kinetic equations for the integral level population. In what follows we employ a similar approach to derive the master equation for multi-electron distribution functions. This becomes possible since the transitions between multi-electron states are determined by one-particle transition operators (5) and (8), while the Coulomb interaction, taken in the form (25), conserves the number of electrons occupying the precise MOs and thus does not lead to electron-electron transitions.

It has been shown earlier in [26] that the occupation number representation offers a convenient way to derive kinetic equations in a multi-electron transfer system. Here, we use the same representation and suppose that the singleelectron states as well as the corresponding single-electron energies are already known. Thus, each single-electron state $\left|j \sigma_{j}\right\rangle(j=L \mathbf{k}, R \mathbf{q}, f)$ is associated with the occupation number state $\left|N_{j \sigma_{j}}\right\rangle$ at $N_{j \sigma_{j}}=1$. [Remember that $N_{j \sigma_{j}}=0,1$ is the number of electrons occupying the single-electron state $j$ with spin-projection $\sigma_{j}$.] Choosing the Coulomb interaction in the form (25) we are able to represent multi-electron states (27) as the product of single-electron states. Because the states (27) are diagonal with respect to the occupation numbers the diagonal elements $\mathscr{P}_{a}(t)=\langle a|\rho(t)| a\rangle$ of the LMR density operator $\rho(t)$ can be derived from relation

$$
\mathscr{P}_{a}(t)=\left\langle a\left|\rho_{d}(t)\right| a\right\rangle=\left\langle\{N\} v\left(\left\{N_{f \sigma_{f}}\right\}\right)\left|\rho_{d}(t)\right|\{N\} v\left(\left\{N_{f \sigma_{f}}\right\}\right)\right\rangle,
$$

where $\rho_{d}(t)=\hat{T}_{d} \rho(t)$ is a diagonal density matrix of the LMR system. The time-evolution of the latter is defined by the master Eq. (A.14) but now written in a multi-electron basis. Therefore, the matrix form of the corresponding master equation coincides with the following set of linear equations for the multi-electron populations $\mathscr{P}_{a}(t)$ (see also [26]),

$$
\dot{\mathscr{P}}_{a}(t)=-\sum_{b}\langle a|(\mathscr{Q}|b\rangle\langle b|)| a\rangle \mathscr{P}_{b}(t) .
$$

It is very important that the form of the superoperator 2 is given by the same expansion (A.15) and (A.16) where, however, the single-electron states $|s \alpha\rangle$ have been replaced the multi-electron states (27). [This follows from the fact that we consider only single-electron transitions caused by the same one-particle operator $V_{\text {int }}=V_{M-M}+V_{L R-M}$ (cf. Eqs. (5) and (8)).]

Now we take into consideration the two following points. The first is related to the form of LMR states (27) which appear as a product of single-particle states. It means that one can represent a many-particle population $\mathscr{P}_{a}(t)$ as a product of single-particle populations,

$$
\mathscr{P}_{a}(t)=\prod_{\mathbf{k} \sigma_{\mathbf{k}}} P\left(N_{L \mathbf{k} \sigma_{\mathbf{k}}}, t\right) \prod_{\mathbf{q} \sigma_{\mathbf{q}}} P\left(N_{R \mathbf{q} \sigma_{\mathbf{q}}}, t\right) \prod_{f \sigma_{f}} P\left(N_{f \sigma_{f}} v\left(\left\{N_{f \sigma_{f}}\right\}\right), t\right) .
$$

Here, the quantities $P\left(N_{j \sigma_{j}}, t\right)$ define the probability that a transferred excess electron with spin quantum number $\sigma_{j}$ does $\left(N_{j \sigma_{j}}=1\right)$ or does not $\left(N_{j \sigma_{j}}=0\right)$ occupy a single-electron lead state $j=L \mathbf{k}, R \mathbf{q}$. The probabilities $P\left(N_{f \sigma_{f}} v\left(\left\{N_{f \sigma_{f}}\right\}\right), t\right)$ indicate that an excess electron with spin quantum number $\sigma_{f}$ does or does not occupy the $v\left(\left\{N_{f \sigma_{f}}\right\}\right)$ th vibrational level belonging to the $f$ th molecular electronic level. The second point notices the fast intrastate relaxation. Accordingly, the probabilities $P\left(N_{f \sigma_{f}} v\left(\left\{N_{f \sigma_{f}}\right\}\right), t\right)$ can be expressed by the integral populations

$$
P\left(N_{f \sigma_{f}}, t\right)=\sum_{v\left(\left\{N_{f \sigma_{f}}\right\}\right)} P\left(N_{f \sigma_{f}} v\left(\left\{N_{f \sigma_{f}}\right\}\right), t\right)
$$

in noting the relation

$$
P\left(N_{f \sigma_{f}} v\left(\left\{N_{f \sigma_{f}}\right\}\right), t\right)=W\left(E\left(v\left(\left\{N_{f \sigma_{f}}\right\}\right)\right)\right) P\left(N_{f \sigma_{f}}, t\right)
$$

where the probability to populate the vibrational state $v\left(\left\{N_{f \sigma_{f}}\right\}\right)$ reads

$$
W\left(E\left(v\left(\left\{N_{f \sigma_{f}}\right\}\right)\right)\right)=W\left(\epsilon_{f \alpha_{f}}\right) N_{f \sigma_{f}}+W\left(\epsilon_{\lambda}^{(0)}\right)\left(1-N_{f \sigma_{f}}\right) .
$$

If $N_{f \sigma_{f}}=1$ then $P\left(1_{f \sigma_{f}}, t\right)$ coincides with the population $P_{f \sigma_{f}}(t)$ of the $f$ th $\mathrm{MO}$. Therefore, the weights $W\left(E\left(v\left(\left\{1_{f \sigma_{f}}\right\}\right)\right)\right)=W\left(\epsilon_{f \alpha_{f}}\right)$ are identical with those given by the relation (A.6). For $N_{f \sigma_{f}}=0$ the weights $W\left(E\left(v\left(\left\{0_{f \sigma_{f}}\right\}\right)\right)\right)=W\left(\epsilon_{\lambda}^{(0)}\right)$ are defined by Eq. (A.27).

In line with Eq. (31) the multi-electron population $\mathscr{P}_{a}(t)=\mathscr{P}\left(\{N\} v\left(\left\{N_{f \sigma_{f}}\right\}, t\right)\right.$ is expressed by its integral population $\mathscr{P}_{\{N\}}(t)=\sum_{v\left(\left\{N_{\left.f \sigma_{f}\right\}}\right\}\right.} \mathscr{P}_{a}(t)$ via the same relation (A.5) that exists between molecular populations $P\left(N_{f \sigma_{f}}, t\right)$ and $P\left(N_{f \sigma_{f}} v\left(\left\{N_{f \sigma_{f}}\right\}\right), t\right)$. This circumstance along with the fact that in our case the operator $V_{\text {int }}$ is identical with the sum off-diagonal operators (5) and (8), allows us to reduce the basic equations (30) to the balance-like equations of the multi-electron populations 


$$
\dot{\mathscr{P}}_{\{N\}}(t)=-\sum_{\left\{N^{\prime}\right\}}\left(K_{\{N\} \rightarrow\left\{N^{\prime}\right\}} \mathscr{P}_{\{N\}}(t)-K_{\left\{N^{\prime}\right\} \rightarrow\{N\}} \mathscr{P}_{\left\{N^{\prime}\right\}}(t)\right) .
$$

The rate constants

$$
K_{\{N\} \rightarrow\left\{N^{\prime}\right\}}=\frac{2 \pi}{\hbar} \sum_{v\left(\left\{N_{f \sigma_{f}}\right\}\right)} \sum_{v\left(\left\{N_{f \sigma_{f}}^{\prime}\right\}\right.} \prod_{f \sigma_{f}} W\left(E\left(v\left(\left\{N_{f \sigma_{f}}\right\}\right)\right)\left|V\left(\{N\} v\left(\left\{N_{f \sigma_{f}}\right\}\right),\left\{N^{\prime}\right\} v\left(\left\{N_{f \sigma_{f}}^{\prime}\right\}\right)\right)\right|^{2} L\left(E_{a}-E_{b}\right)\right.
$$

characterize a transition from the multi-electron state $|\{N\}\rangle=\prod_{\mathbf{k} \sigma_{\mathbf{k}}}\left|N_{L \mathbf{k} \sigma_{\mathbf{k}}}\right\rangle \times \prod_{\mathbf{q} \sigma_{\mathbf{q}}}\left|N_{R \mathbf{q} \sigma_{\mathbf{q}}}\right\rangle \prod_{f \sigma_{f}}\left|N_{f \sigma_{f}}\right\rangle$ to the state $\left|\left\{N^{\prime}\right\}\right\rangle=\prod_{\mathbf{k} \sigma_{\mathbf{k}}}\left|N_{L \mathbf{k} \sigma_{\mathbf{k}}}^{\prime}\right\rangle \prod_{\mathbf{q} \sigma_{\mathbf{q}}}\left|N_{R \mathbf{q} \sigma_{\mathbf{q}}}^{\prime}\right\rangle \times \prod_{f \sigma_{f}}\left|N_{f \sigma_{f}}^{\prime}\right\rangle$. In Eq. (36), the summation covers all vibrational states related to the molecular levels involved in the electron transmission. Quantity $L\left(E_{a}-E_{b}\right)=(1 / \pi)\left[\left(\gamma_{a}+\gamma_{b}\right) /\left(\left(E_{a}-E_{b}\right)^{2}+\left(\gamma_{a}+\gamma_{b}\right)^{2}\right)\right]$ is the normalized Lorentzian with $\gamma_{a}$ and $\gamma_{b}$ being the total broadenings of single-electron levels participating in a given $|a\rangle \rightarrow|b\rangle$ transition (see below). Note that $L\left(E_{a}-E_{b}\right)$ reduces to a delta-function $\delta\left(E_{a}-E_{b}\right)$ if $\gamma_{a} \rightarrow 0$ and $\gamma_{b} \rightarrow 0$. Such a situation appears if an elastic lead-to-lead electron transmission occurs in the LMR system. The energy $E_{a}=E\left(\{N\}, v\left(\left\{N_{f \sigma_{f}}\right\}\right)\right)$ of the ath multi-electron state reads

$$
E_{a}=\sum_{\mathbf{k} \sigma_{\mathbf{k}}} E_{L \mathbf{k}} N_{L \mathbf{k} \sigma_{\mathbf{k}}}+\sum_{\mathbf{q} \sigma_{\mathbf{q}}} E_{R \mathbf{q}} N_{R \mathbf{q} \sigma_{\mathbf{q}}}+\sum_{f \sigma_{f}}\left(\epsilon_{f \alpha_{f}}-\varepsilon_{\lambda}^{(0)}\right) N_{f \sigma_{f}}+U(\{N\}) .
$$

It contains the contributions of single-electron energies $E_{L \mathbf{k}}, E_{R \mathbf{q}}$ and $\epsilon_{f \alpha_{f}}=\operatorname{Re} \mathscr{E}_{f \alpha_{f}}$ as well as the term $U(\{N\})$ including the interaction between the extra electrons when the latter populate the molecule in the course of charge transmission. The energy $E_{b}$ is defined by the same form (37) (but with the replacement of the set $\{N\}$ by the set $\left\{N^{\prime}\right\}$ ). To calculate the matrix elements $V\left(\{N\} v\left(\left\{N_{f \sigma_{f}}\right\}\right),\left\{N^{\prime}\right\} v\left(\left\{N_{f \sigma_{f}}^{\prime}\right\}\right)\right)=\left\langle\{N\} v\left(\left\{N_{f \sigma_{f}}\right\}\right)\left|V^{(\text {eff })}\right|\left\{N^{\prime}\right\} v\left(\left\{N_{f \sigma_{f}}\right\}\right)\right\rangle$ one has to employ the effective operator (A.20) where $V_{\text {int }}$ is the off-diagonal transfer operator and $G(E)=(E-H)^{-1}$ is Green's operator of the total LMR system $\left(H=H_{L M R}^{(\text {eff })}\right.$ is the effective Hamiltonian of the LMR system, Eq. (28), and $E=E_{a} \approx E_{b}$ is the transition energy, respectively). Note again that if only single-electron transitions are responsible for electron transmission then $V_{\text {int }}=V_{M-M}+V_{L R-M}$.

\subsection{Kinetic equations for the reduced single-electron distribution functions}

Noting the linear balance-like set of equations (35) for multi-electron distribution functions the kinetic equations for the $P_{j \sigma_{j}}(t)$ follow in using the relations (13) and (14). Furthermore, we introduce the ansatz (31) into kinetic equations (35), sum up both parts of Eq. (35) over complete set of initial $(\{N\})$ and final $\left(\left\{N^{\prime}\right\}\right)$ occupation numbers, and, finally apply the normalization condition (15). This all yields the following set of nonlinear kinetic equations for the singleelectron populations

$$
\dot{P}_{j \sigma_{j}}(t)=\sum_{\{N\}} \sum_{\left\{N^{\prime}\right\}} \prod_{\mathbf{k} \sigma_{\mathbf{k}}} \prod_{\mathbf{q} \sigma_{\mathbf{q}}} \prod_{f \sigma_{f}} N_{j \sigma_{j}}\left[K_{\{N\} \rightarrow\left\{N^{\prime}\right\}} P\left(N_{L \mathbf{k} \sigma_{\mathbf{k}}}, t\right) P\left(N_{R \mathbf{q} \sigma_{\mathbf{q}}}, t\right) \times P\left(N_{f \sigma_{f}}, t\right)-K_{\left\{N^{\prime}\right\} \rightarrow\{N\}} P\left(N_{L \mathbf{k} \sigma_{\mathbf{k}}}^{\prime}, t\right) P\left(N_{R \mathbf{q} \sigma_{\mathbf{q}}}^{\prime}, t\right) P\left(N_{f \sigma_{f}}^{\prime}, t\right)\right] .
$$

The nonlinearity is caused by the Coulomb interaction among different transferred electrons. It influences the transfer rates $K_{\{N\} \rightarrow\left\{N^{\prime}\right\}}$, Eq. (36) via the energy differences $E_{a}-E_{b}$ between the multi-electron states $|a\rangle=|\{N\}\rangle$ and $|b\rangle=\left|\left\{N^{\prime}\right\}\right\rangle$. According to Eq. (37) the $E_{a}-E_{b}$ may be specified to the molecular energy $\epsilon_{f \alpha}$ together with a Coulomb term.

Moreover, condition (18) allows to represent the energy difference $E_{a}-E_{b}$ between two multi-electron states in the form (cf. Eq. (37))

$$
E_{a}-E_{b} \approx \sum_{\mathbf{k}, \sigma_{\mathbf{k}}} E_{L \mathbf{k}}\left(N_{L \mathbf{k} \sigma_{\mathbf{k}}}-N_{L \mathbf{k} \sigma_{\mathbf{k}}}^{\prime}\right)+\sum_{\mathbf{q}, \sigma_{\mathbf{q}}} E_{R \mathbf{q}}\left(N_{R \mathbf{q} \sigma_{\mathbf{q}}}-N_{R \mathbf{q} \sigma_{\mathbf{q}}}^{\prime}\right)+\sum_{\mu \sigma_{\mu}}\left(\varepsilon_{\mu \alpha_{\mu}}-\varepsilon_{\lambda}^{(0)}\right)\left(N_{\mu \sigma_{\mu}}-N_{\mu \sigma_{\mu}}^{\prime}\right)+\left(U(\{N\})-U\left(\left\{N^{\prime}\right\}\right)\right) .
$$

Eqs. (39) and (26) define the general expressions for the energy difference between multi-electron states $|a\rangle=|\{N\}\rangle$ and $|b\rangle=\left|\left\{N^{\prime}\right\}\right\rangle$ which are involved in the $a \rightarrow b$-transition.

\subsection{Single-electron transmission channels}

For the transfer problem under consideration respective transitions are described by the operators (5) and (8). Therefore, each elementary electron-transfer step connects only two precise electronic states. This means that only 
two states change their electronic population while that of the remaining states remains unchanged. Thus, in the case of lead-molecule transfer the corresponding transition matrix element follows as

$$
\begin{aligned}
& V_{\left\{N^{\prime}\right\}\{N\}}=\sum_{\mathbf{k} \sigma_{\mathbf{k}}} \sum_{\mu \sigma_{\mu}}\left[V_{L \mathbf{k} \lambda, \mu \alpha_{\mu}} N_{L \mathbf{k} \sigma_{\mathbf{k}}}\left(1-N_{\mu \sigma_{\mu}}\right)+V_{L \mathbf{k} \lambda, \mu \alpha_{\mu}}^{*} N_{L \mathbf{k} \sigma_{\mathbf{k}}}^{\prime}\left(1-N_{\mu \sigma_{\mu}}^{\prime}\right)\right] \\
& \times \delta_{\sigma_{\mathbf{k}}, \sigma_{\mu}} \delta_{N_{\mu \sigma_{\mu}}^{\prime}, 1-N_{\mu \sigma}} \delta_{N_{L \mathbf{k} \sigma_{\mathbf{k}}}^{\prime}, 1-N_{L \mathbf{k} \sigma_{\mathbf{k}}}} \prod_{\mathbf{k}^{\prime} \sigma_{\mathbf{k}^{\prime}} \neq \mathbf{k} \sigma_{\mathbf{k}}} \delta_{N_{L \mathbf{k}^{\prime} \sigma_{\mathbf{k}^{\prime}}}^{\prime}, N_{L \mathbf{k}^{\prime} \boldsymbol{k}^{\prime}}} \prod_{\mu^{\prime} \sigma_{\mu^{\prime}} \neq \mu \sigma_{\mu}} \delta_{N_{\mu^{\prime} \sigma_{\mu^{\prime}}}, N_{\mu^{\prime} \sigma_{\mu^{\prime}}}} \prod_{\mathbf{q} \sigma_{\mathbf{q}}} \delta_{N_{R \mathbf{q} \sigma_{\mathbf{q}}}^{\prime}, N_{R \mathbf{q}} \sigma_{\mathbf{q}}} .
\end{aligned}
$$

In the case of a transition between extended molecular states one may derive

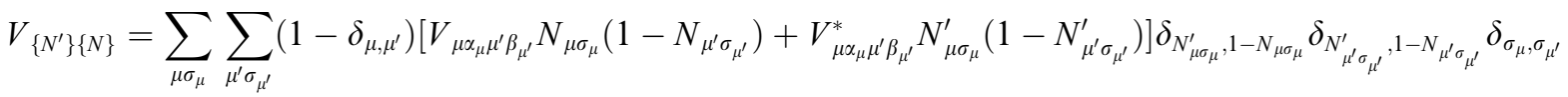

$$
\begin{aligned}
& \times \prod_{\mathbf{k} \sigma_{\mathbf{k}}} \delta_{N_{L \mathbf{k} \sigma_{\mathbf{k}}}^{\prime}, N_{L \mathbf{k} \sigma_{\mathbf{k}}}} \prod_{\mu^{\prime} \sigma_{\mu^{\prime}} \neq \mu \sigma_{\mu}} \delta_{N_{\mu^{\prime}} \sigma_{\mu^{\prime}}}, N_{\mu^{\prime} \sigma_{\mu^{\prime}}} \prod_{\mathbf{q} \sigma_{\mathbf{q}}} \delta_{N_{R \mathbf{q} \sigma}^{\prime}, N_{R \mathbf{q}} \sigma_{\mathbf{q}}} .
\end{aligned}
$$

The matrix element $T_{\left\{N^{\prime}\right\}\{N\}}$ which characterizes electron transitions between the two leads is calculated in using the second term of the effective operator (A.20) written in the occupation number representation. As it has been shown in part A.3 of the Appendix, the calculation of the single-electron lead-lead matrix element, Eq. (A.33) can be carried out in using the simple form (A.35). This follows from the fact that Green function $G(E)$ becomes diagonal if the electron-vibrational Hamiltonian $H=H_{L M R}^{(\mathrm{e}-\mathrm{v})}$, Eq. (A.23) of the complete LMR system is replaced by an effective electronvibrational Hamiltonian (17) with (complex) eigenvalues $\mathscr{E}_{f \alpha_{f}}$. [Note that single-electron Hamiltonian $H_{L M R}^{(\mathrm{e}-\mathrm{v})}$ represents particular version of the multi-electron Hamiltonian $H_{L R M}^{\text {(eff) }}$; compare Eqs. (A.23) and (25).]

If one and more excess electrons are already captured by the molecule the transmission of an additional electron through the molecule occurs against the background of a Coulomb interaction between the transferred electrons. It means that Green function $G(E)=[E-H]^{-1}$ has to be calculated with the effective LMR Hamiltonian $H=H_{L R M}^{\text {(eff) }}$ which contains the noted Coulomb interaction. Taking this circumstance into consideration and choosing the Coulomb interaction in the form (25), it becomes obvious that the effective Hamiltonian of the LMR system, Eq. (28) is diagonal in the occupation number representation, i.e. it reads $H=H_{L M R}^{\text {(eff })}=\sum_{\{N\}} E_{\{N\}}|\{N\}\rangle\langle\{N\}|$, where the multi-electron energy reads

$$
E_{\{N\}}=\sum_{r=L, R} \sum_{\mathbf{k} \sigma_{\mathbf{k}}} E_{r \mathbf{k}} N_{r \mathbf{k} \sigma_{\mathbf{k}}}+\sum_{\mu \sigma_{\mu}}\left(\mathscr{E}_{\mu \alpha_{\mu}}-\varepsilon_{\lambda}^{(0)}\right) N_{\mu \sigma_{\mu}}+U \sum_{\mu, \mu^{\prime}} \Phi\left(\mu \mu ; \mu^{\prime} \mu^{\prime}\right) N_{\mu \uparrow} N_{\mu^{\prime} \downarrow}
$$

Note that this expression accounts for fast relaxational transitions as well as for the influence of the leads on the molecular levels via the single-electron energies $\mathscr{E}_{\mu \alpha_{\mu}}$, Eq. (19). As far as the Hamiltonian of the LMR system is diagonal, Green's function in the transition operator (A.20) is diagonal as well. We may write

$$
T_{\left\{N^{\prime}\right\}\{N\}}=\left\langle\left\{N^{\prime}\right\}\left|V_{\text {int }} G(E) V_{\text {int }}\right|\{N\}\right\rangle=\sum_{\left\{N^{\prime \prime}\right\}} V_{\left\{N^{\prime}\right\}\left\{N^{\prime \prime}\right\}} G_{\left\{N^{\prime \prime}\right\}\left\{N^{\prime \prime}\right\}}(E) V_{\left\{N^{\prime \prime}\right\}\{N\}},
$$

where the matrix elements $V_{\left\{N^{\prime}\right\}\left\{N^{\prime \prime}\right\}}$ and $V_{\left\{N^{\prime \prime}\right\}\{N\}}$ are given by Eq. (40), while we get $\left(E=E_{\{N\}} \approx E_{\left\{N^{\prime}\right\}}\right)$

$$
\begin{aligned}
G_{\left\{N^{\prime \prime}\right\}\left\{N^{\prime \prime}\right\}}(E) & =\left\langle\left\{N^{\prime \prime}\right\}|G(E)|\left\{N^{\prime \prime}\right\}\right\rangle=\left[E_{\{N\}}-E_{\left\{N^{\prime \prime}\right\}}\right]^{-1} \\
& =\left[\sum_{\mathbf{k} \sigma_{\mathbf{k}}} E_{L \mathbf{k}}\left(N_{L \mathbf{k} \sigma_{\mathbf{k}}}-N_{L \mathbf{k} \sigma_{\mathbf{k}}}^{\prime \prime}\right)+\sum_{\mu \sigma_{\mu}}\left(\mathscr{E}_{\mu \alpha_{\mu}}-\varepsilon_{\lambda}^{(0)}\right)\left(N_{\mu \sigma_{\mu}}-N_{\mu \sigma_{\mu}}^{\prime \prime}\right)+U \sum_{\mu \nu} \Phi(\mu \mu, \nu v)\left(N_{\mu \uparrow} N_{v \downarrow}-N_{\mu \uparrow}^{\prime \prime} N_{v \downarrow}^{\prime \prime}\right)\right]^{-1} .
\end{aligned}
$$

Note that energy $\mathscr{E}_{\mu \alpha_{\mu}}$ includes the influence of the leads on the single-electron molecular energies while.

One can see that two different types of electronic pathways participate in the formation of the coupling $T_{\left\{N^{\prime}\right\}\{N\}}$. The first is associated with the same state $\mu \sigma_{\mu}$ connecting the two lead states, $L \mathbf{k} \sigma_{\mathbf{k}}$ and $R \mathbf{q} \sigma_{\mathbf{q}}$. For instance, if an electron is transferred from the left lead to the right lead then

$$
\begin{aligned}
& T_{\left\{N^{\prime}\right\}\{N\}}=\sum_{\left\{N^{\prime \prime}\right\}} \sum_{\mathbf{k} \sigma_{\mathbf{k}}} \sum_{\mathbf{q} \sigma_{\mathbf{q}}} \sum_{\mu \sigma_{\mu}} V_{L \mathbf{k} \lambda, \mu \alpha_{\mu}}^{*} V_{R \mathbf{q} \lambda^{\prime}, \mu \beta_{\mu}}\left[E_{\{N\}}-E_{\left\{N^{\prime \prime}\right\}}\right]^{-1} N_{L \mathbf{k} \sigma_{\mathbf{k}}}\left(1-N_{\mu \sigma_{\mu}}\right)\left(1-N_{R \mathbf{q} \sigma_{\mathbf{q}}}\right) \\
& \times \delta_{\sigma_{\mathbf{k}}, \sigma_{\mu}} \delta_{\sigma_{\mu}, \sigma_{\mathbf{q}}} \delta_{N_{L \mathbf{k} \sigma_{\mathbf{k}}}^{\prime \prime}, 1-N_{L \mathbf{k} \sigma_{\mathbf{k}}}} \delta_{N_{L \mathbf{k} \sigma_{\mathbf{k}}}^{\prime}, 1-N_{L \mathbf{k} \sigma_{\mathbf{k}}}} \delta_{N_{\mu \sigma_{\mu}}^{\prime \prime}, 1-N_{\mu \sigma_{\mu}}} \delta_{N_{\mu \sigma \mu}^{\prime}, N_{\mu \sigma_{\mu}}} \delta_{N_{R \mathbf{q} \sigma_{\mathbf{q}}}^{\prime \prime}, N_{R q} \sigma_{\mathbf{q}}} \delta_{N_{R \mathbf{q} \sigma_{q}}^{\prime}, 1-N_{R q} \sigma_{q}}
\end{aligned}
$$

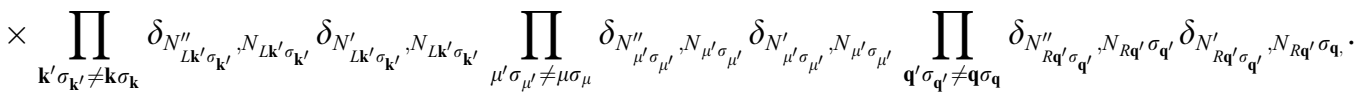


It can be seen that the coupling $T_{\left\{N^{\prime}\right\}\{N\}}$ is formed in such a manner that it contains the sum of terms each being proportional to the factor $N_{L \mathbf{k} \sigma_{\mathbf{k}}}\left(1-N_{\mu \sigma_{\mu}}\right)\left(1-N_{R \mathbf{q} \sigma_{\mathbf{q}}}\right)$. The conservation of electron spin during transition $\left(\sigma_{\mathbf{k}}=\sigma_{\mu}=\sigma_{\mathbf{q}}\right)$ results in a specific dependence of $T_{\left\{N^{\prime}\right\}\{N\}}$ on the occupation numbers.

Fig. 2 displays the elastic electron transmission from the $L \mathbf{k}$ th level of the left lead to the Rqth level of the right lead through an "empty" molecule as well as a molecule containing a single excess electron at a certain $\mu$ 'th MO. To let become the picture more clear the molecular vibronic levels are not represented in the schemes (a) and (b). This means that instead of actual initial, intermediate and final LMR energies $\left(E_{L \mathbf{k}}+\varepsilon_{\lambda}^{(0)}, \mathscr{E}_{\mu \alpha_{\mu}}\right.$ and $E_{R \mathbf{q}}+\varepsilon_{\lambda}^{(0)}$, respectively) only single-electron energies, $E_{L \mathbf{k}}, \varepsilon_{\mu}$ and $E_{R \mathbf{q}}$ are displayed. Correspondingly, only electronic couplings, $V_{L \mathbf{k}}$ and $V_{R \mathbf{q}}$ indicate the transitions between single-electron levels instead of the electron-vibration couplings (9). Note, that just these couplings to the intermediate state connect the initial and final states. Since each separate single-electron transition does not influence the spin-projection of the transferred electron (cf. Eq. (8)), the transmission of an electron through an "empty" molecule occurs without any change of spin-projection of the transferred electron so that $\sigma_{\mathbf{k}}=\sigma_{\mu}=\sigma_{\mathbf{q}}$. A similar spin conservation is also valid during the transmission through a molecule which already contains an excess electron at a certain $\mu^{\prime}$ th $\mathrm{MO}$ provided that the excess electron does not leave the molecule during the transmission process (cf. scheme (b) in Fig. 2).

The second type of pathway covers two different states $\mu \sigma_{\mu}$ and $\mu^{\prime} \sigma_{\mu^{\prime}}$ in single-electron transmission. Therefore,

$$
\begin{aligned}
& T_{\left\{N^{\prime}\right\}\{N\}}=\sum_{\left\{N^{\prime \prime}\right\}} \sum_{\mathbf{k} \sigma_{\mathbf{k}}} \sum_{\mathbf{q} \sigma_{\mathbf{q}}} \sum_{\mu \sigma_{\mu}} \sum_{\mu^{\prime} \sigma_{\mu^{\prime}}} V_{L \mathbf{k} \lambda, \mu \alpha_{\mu}}^{*} V_{R \mathbf{q} \lambda^{\prime}, \mu^{\prime} \beta_{\mu^{\prime}}}\left[E_{\{N\}}-E_{\left\{N^{\prime \prime}\right\}}\right]^{-1} N_{L \mathbf{k} \sigma_{\mathbf{k}}}\left(1-N_{\mu \sigma_{\mu}}\right) N_{\mu^{\prime} \sigma_{\mu^{\prime}}}\left(1-N_{R \mathbf{q} \sigma_{\mathbf{q}}}\right)\left(1-\delta_{\mu \sigma_{\mu}, \mu^{\prime} \sigma_{\mu^{\prime}}}\right)
\end{aligned}
$$

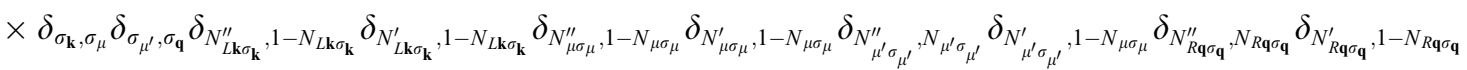

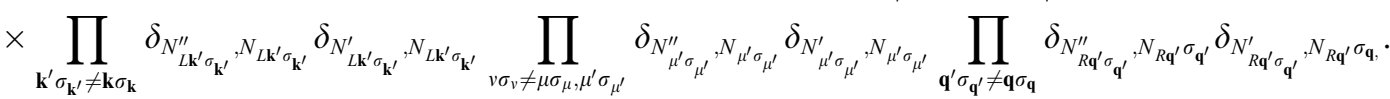

This expression shows that the second type of transmission pathway contains the sum of terms each being proportional to the factors $N_{L \mathbf{k} \sigma_{\mathbf{k}}}\left(1-N_{\mu \sigma_{\mu}}\right) N_{\mu^{\prime} \sigma_{\mu^{\prime}}}\left(1-N_{R \mathbf{q} \sigma_{\mathbf{q}}}\right)$. Spin conservation results in $\sigma_{\mathbf{k}}=\sigma_{\mu}, \sigma_{\mu^{\prime}}=\sigma_{\mathbf{q}}$. The second type of pathway may work even through one and the same extended MO, $\mu^{\prime}=\mu$. But, in contrast to the first type the transmission becomes only possible if $\sigma_{\mu^{\prime}} \neq \sigma_{\mu}$ (at $\mu^{\prime} \neq \mu$ ) or $\sigma_{\mu}^{\prime} \neq \sigma_{\mu}$ (at $\mu^{\prime}=\mu$ ). Fig. 3 displays a transfer process where the $\mu$ th molecular level (preliminary occupied by an excess electron) controls the transmission. It is seen that in this case the spin projection of the incoming (into the molecule) and the outgoing (out of the molecule) electron can differ (compare the schemes (a) and (b)).
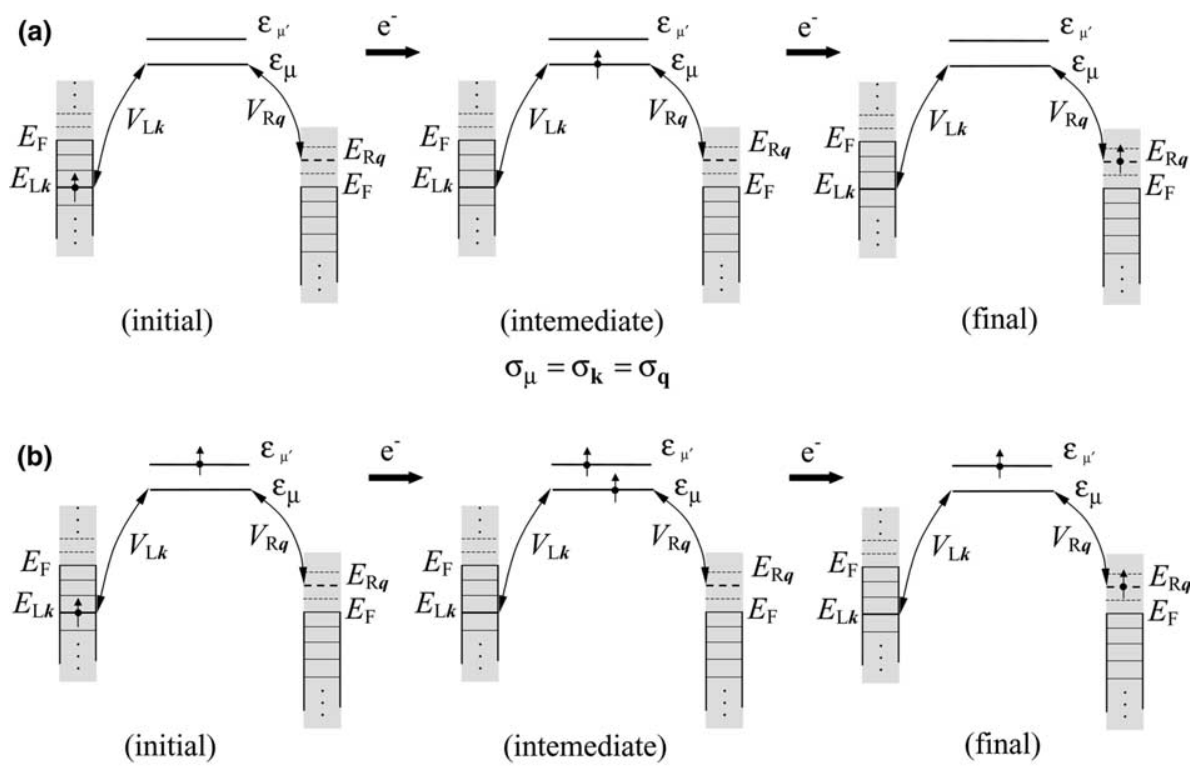

$$
\sigma_{\mu}=\sigma_{\mathbf{k}}=\sigma_{\mathbf{q}}
$$

Fig. 2. Single-electron scheme of electron transmission through an "empty" (a) and singly occupied (b) molecule. Only single-electron levels with energies $E_{L \mathbf{k}}, \varepsilon_{\mu}$, and $E_{R \mathbf{q}}$ are represented. Transitions between the corresponding electronic states are characterized by couplings $V_{L \mathbf{k}}$ and $V_{R \mathbf{q}}$. The LMR intermediate state is characterized by the presence of one or two excess electrons. The transmission does not change a spin state of the transmitted electron, $\sigma_{\mathbf{k}}=\sigma_{\mu}=\sigma_{\mathbf{q}}$. 

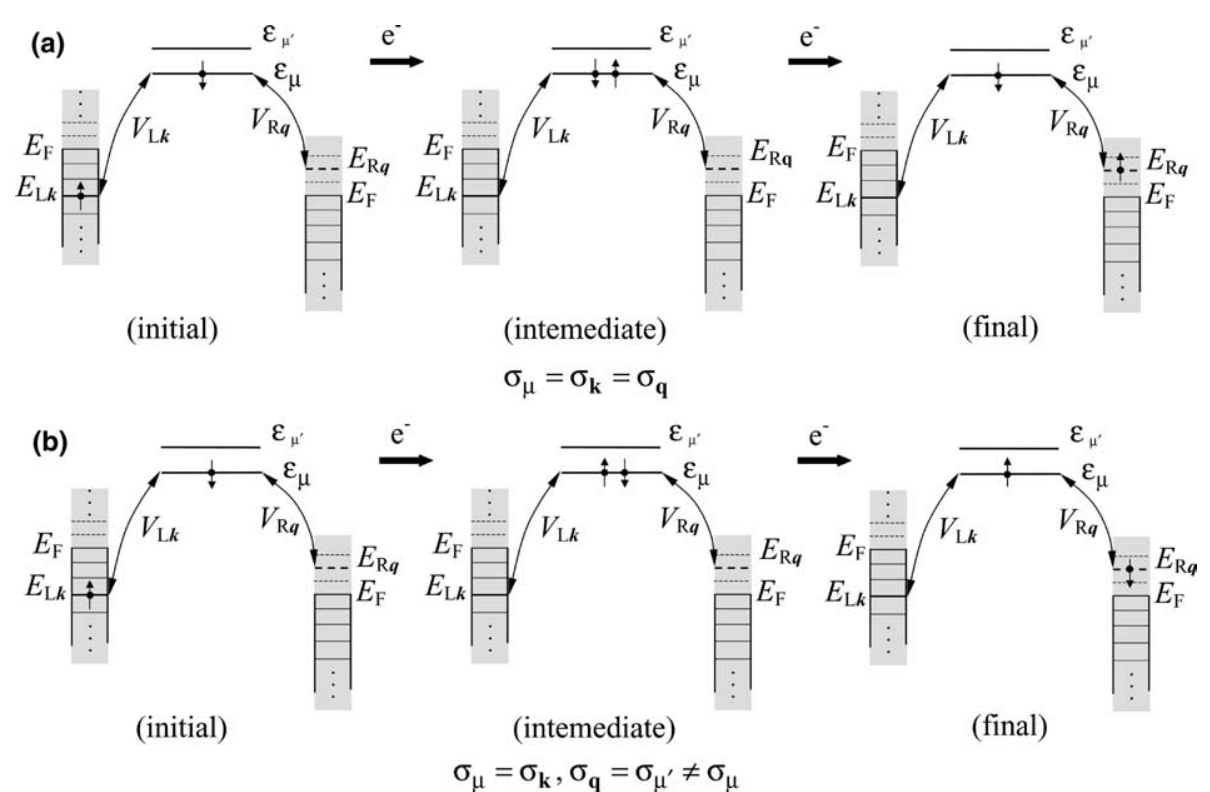

Fig. 3. Electron transmission through a singly occupied molecular level $\mu$. The spin of the transmitted electron is either conserved or changed.

Now we are able to write down the precise form of transfer rates (36) specifying the kinetic equations (38). As an example, let us derive the transfer rate which characterizes an electron jump from the left lead into the molecule. Such a hopping transition occurs from the set of electronic lead states $L \mathbf{k} \sigma_{\mathbf{k}}$ to the set of extended molecular states $\mu \sigma_{\mu}$. During the transfer of an electron from a particular lead state $L \mathbf{k} \sigma_{\mathbf{k}}$ to the molecular level $\mu \sigma_{\mu}$ the occupation numbers changes in such a way that $N_{L \mathbf{k} \sigma_{\mathbf{k}}} \rightarrow N_{L \mathbf{k} \sigma_{\mathbf{k}}}^{\prime}=1-N_{L \mathbf{k} \sigma_{\mathbf{k}}}$ and $N_{\mu \sigma_{\mu}} \rightarrow N_{\mu \sigma_{\mu}}^{\prime}=1-N_{\mu \sigma_{\mu}}$. In line with Eqs. (39), (26) and (40) it yields

$$
K_{\left\{N^{\prime}\right\}\{N\}}=\sum_{\left\{N_{v}\right\}} \sum_{\mathbf{k} \sigma_{\mathbf{k}}} \sum_{\mu \sigma_{\mu}} K_{\left\{N_{\mu}\right\}\left\{N_{v}\right\}}\left(L \mathbf{k} \sigma_{\mathbf{k}} \rightarrow \mu \sigma_{\mu}\right) .
$$

The summation covers all possible lead-molecule electron transitions each being characterized by partial transfer rate

$$
K_{\left\{N_{\mu}\right\}\left\{N_{v}\right\}}\left(L \mathbf{k} \sigma_{\mathbf{k}} \rightarrow \mu \sigma_{\mu}\right)=\delta_{\sigma_{\mathbf{k}}, \sigma_{\mu}} \frac{2 \pi}{\hbar}\left|V_{L \mathbf{k}}\right|^{2} N_{L \mathbf{k} \sigma_{\mathbf{k}}}\left(1-N_{\mu \sigma_{\mu}}\right)(\mathrm{FC})_{\left\{N_{\mu}\right\}\left\{N_{v}\right\}}\left(L \mathbf{k} \sigma_{\mathbf{k}} \rightarrow \mu \sigma_{\mu}\right) .
$$

Here, the rate depends on the population of the molecule by the preliminary captured electrons. The latter are accounted for by the set of occupation numbers $\left\{N_{v}\right\}$ which all enter the Franck-Condon factor

$$
(\mathrm{FC})_{\left\{N_{\mu}\right\}\left\{N_{v}\right\}}\left(L \mathbf{k} \sigma_{\mathbf{k}} \rightarrow \mu \sigma_{\mu}\right)=\frac{1}{\pi} \sum_{\lambda \alpha_{\mu}} W\left(\varepsilon_{\lambda}^{(0)}\right) \frac{\left.\left|u_{\mu}(1)\right|^{2}\left\langle\chi_{\lambda} \mid \chi_{\mu \alpha_{\mu}}\right\rangle\right|^{2}\left(\gamma_{\lambda}+\Gamma_{\mu \alpha_{\mu}}\right)}{\Delta E_{\left\{N_{\mu}\right\}\left\{N_{v}\right\}}^{2}\left(L \mathbf{k} \sigma_{\mathbf{k}} \rightarrow \mu \sigma_{\mu}\right)+\left(\gamma_{\lambda}+\Gamma_{\mu \alpha_{\mu}}\right)^{2}}
$$

via the energy difference

$$
\Delta E_{\left\{N_{\mu}\right\}\left\{N_{v}\right\}}\left(L \mathbf{k} \sigma_{\mathbf{k}} \rightarrow \mu \sigma_{\mu}\right)=E_{L \mathbf{k}}-\left(\epsilon_{\mu \alpha_{\mu}}-\varepsilon_{\lambda}^{(0)}\right)+U \sum_{v} \Phi(\mu \mu ; \nu v)\left(N_{\mu \sigma_{\mu}}-N_{\mu \sigma_{\mu}}^{\prime}\right)\left(N_{v \downarrow} \delta_{\sigma_{\mu} \uparrow}+N_{v \uparrow} \delta_{\sigma_{\mu} \downarrow}\right) .
$$

In accordance with Eq. (48) the transfer only takes place if $N_{\mu \sigma_{\mu}}=0, N_{\mu \sigma_{\mu}}^{\prime}=1$. Therefore, bearing in mind the fact that Franck-Condon factor is maximal if $\Delta E_{\left\{N_{\mu}\right\}\left\{N_{v}\right\}}\left(L \mathbf{k} \sigma_{\mathbf{k}} \rightarrow \mu \sigma_{\mu}\right) \approx 0$, we are led the condition for a resonant lead-molecule transition

$$
E_{L \mathbf{k}}+\varepsilon_{\lambda}^{(0)} \approx \epsilon_{\mu \alpha_{\mu}}+U \sum_{v} \Phi(\mu \mu ; \nu v)\left(N_{v \downarrow} \delta_{\sigma_{\mu}, \uparrow}+N_{v \uparrow} \delta_{\sigma_{\mu}, \downarrow}\right) .
$$

Eqs.(47)-(51) define the completely lead-molecule electron-transfer rate. Analogously, one can specify the lead-lead electron-transfer rate. To this end one has to employ Eqs.(43)-(46) as well as Eq.(36) where the matrix element $V\left(\left\{N^{\prime}\right\} v\left(\left\{N_{f \sigma_{f}}^{\prime}\right\}\right),\{N\} v\left(\left\{N_{f \sigma_{f}}\right\}\right)\right)$ is associated with the $T_{\left\{N^{\prime}\right\}\{N\}}$. It is important to note that the lead-molecule (as well as molecule-lead) and the lead-lead transfer rates appear as the sum of transfer rates related to different transmission channels. The latter are defined by the number of extra electrons preliminary captured by the molecule.In the case of lead-molecule transitions the channels are determined by the occupation numbers $\left\{N_{v}\right\}$. This conclusion follows just 
from Eq. (51). For instance, there exists a transfer channel defined via the condition $N_{v \sigma_{v}}=0$, which is valid for all molecular states $v$ including the $v=\mu, \sigma_{v}=\sigma_{\mu}^{\prime} \neq \sigma_{\mu}$. This channel corresponds to electron jumps from the lead into the molecule provided the molecule does not contain an extra electron. The corresponding channel transfer rate becomes maximal at the resonance condition $E_{L \mathbf{k}}+\varepsilon_{\lambda}^{(0)} \approx \epsilon_{\mu \alpha_{\mu}}$. If a single electron has been already captured by the molecule, the lead-molecule transfer proceeds along another transmission channel. Let for instance an extra electron be captured by the molecule in the state $\mu \sigma_{\mu}^{\prime}$ so that all occupation numbers $N_{v \sigma_{v}}$ are zero except $N_{v \sigma_{v}}=N_{\mu \sigma_{\mu}^{\prime}}=1$ where $\sigma_{\mu}^{\prime} \neq \sigma_{\mu}$. Then, the resonance condition (51) reduces to $E_{L \mathbf{k}}+\varepsilon_{\lambda}^{(0)} \approx \epsilon_{\mu \alpha_{\mu}}+U \Phi(\mu \mu ; \mu \mu)$. It differs essentially from the previous one according to the presence of an additional Coulomb term $U \Phi(\mu \mu ; \mu \mu)$.

Each transmission channel specifies the character of the nonlinearity present in the kinetic equation (38). Thus, if the electron transition proceeds from the lead to the molecule, whereas the latter does not contain an extra electron then the corresponding term on the right-hand side of the kinetic equation (38) includes the product $P_{L \mathbf{k} \sigma_{\mathbf{k}}}(t)\left(1-P_{\mu \sigma_{\mu}}(t)\right)$ $\left(1-P_{\mu \sigma_{\mu}^{\prime}}(t)\right) \prod_{v \sigma_{v} \neq \mu \sigma_{\mu}, \mu \sigma_{\mu}^{\prime}}\left(1-P_{v \sigma_{v}}(t)\right)$. [Note that $P_{j \sigma_{j}}(t)=P\left(1_{j \sigma_{j}}, t\right), 1-P_{j \sigma_{j}}(t)=P\left(0_{j \sigma_{j}}, t\right)$.] If a single extra electron with spin projection $\sigma_{\mu}^{\prime} \neq \sigma_{\mu}$ is already occupies the $\mu$ th $\mathrm{MO}$, the product of state populations reads $P_{L \mathbf{k} \sigma_{\mathbf{k}}}(t)\left(1-P_{\mu \sigma_{\mu}}(t)\right)$ $P_{\mu \sigma_{\mu}^{\prime}}(t) \prod_{v \sigma_{v} \neq \mu \sigma_{\mu}, \mu \sigma_{\mu}^{\prime}}\left(1-P_{v \sigma_{v}}(t)\right)$. In contrast to the foregoing product it contains the population $P_{\mu \sigma_{\mu}^{\prime}}(t)$ instead of $1-P_{\mu \sigma_{\mu}^{\prime}}(t)$. Generally, the precise form of the nonlinear kinetic equation is defined by those types of transmission channels which, at a given voltage bias $V$, provide the major contribution to the formation of the current. In a given LMR system, it strongly depends on the number of MOs involved in the transfer process as well as on the relation between the site-site couplings $V_{m m^{\prime}}$ and the Coulomb interaction.

\section{Restriction on single-electron transmission processes}

The theoretical expressions derived in the preceding section allow us to describe the kinetics of current formation along various types of transmission channels, which differ from each other by the number of excess electrons preliminary captured by the molecule. During the transmission along a single-electron channel the molecule can capture only a single excess electron. Such a channel mainly contributes to the current only for those cases where the Coulombic interaction energy between two excess electrons (captured by the $\mu$ th and $v$ th MOs) exceeds strongly the shift of single-electron energies caused by the applied voltage.

To illustrate this statement we consider a low-temperature single-electron phonon-less transmission through a LUMO-level and formulate the conditions at which such a transmission gives the main contribution to the current. Let $\epsilon_{1}$ be the energetic position of the unbiased LUMO-level in the absence of any Coulombic interaction. We take the Coulomb energy in the form (26) which is valid at a strong inter-site electronic coupling. It means that the energy gaps $\left|\tilde{\epsilon}_{\mu}-\tilde{\epsilon}_{v}\right|$ between the molecular levels satisfy the relation (24) and thus the LUMO-level is assumed to be well separated from the nearest LUMO+1-level. If the left lead is supported at zero voltage then at a strong inter-site coupling each molecular level shows a linear shift (see more details in [79]). This shift is specified by the voltage division factor $\eta$ [55,61], so that the energetic position of the biased LUMO-level is defined through relation $\epsilon_{1}(V)=\epsilon_{1}+\eta e V$. At certain positive values $V=V_{L}^{(\text {lres })}$ where

$$
V_{L}^{(1 \text { res })}=\Delta E_{L}(0) / \eta|e|,
$$

the energy gap $\Delta E_{1 L}(V) \equiv \epsilon_{1}(V)-E_{\mathrm{F}}=\Delta E_{L}(0)-\eta|e| V$ may equal zero. Thus, at $V \geqslant V_{L}^{(1 \text { res })}$ the current is originated by a resonant single-electron transmission through a given energy level. If, however, the LUMO level $\mu=1$ is already occupied by the excess electron then a resonant transmission becomes possible only at $V \geqslant V_{L}^{(2 \text { res })}$ where $V_{L}^{(2 \text { res })}$ is another critical voltage. Here, $V_{L}^{(2 \mathrm{res})}$ is fixed by the condition $\left(2 \epsilon_{1}(V)+U \Phi(11 ; 11)\right)-\left(\epsilon_{1}(V)+E_{\mathrm{F}}\right)=0$, and thus

$$
V_{L}^{(2 \mathrm{res})}=\left(\Delta E_{L}(0)+U \Phi(11 ; 11) / \eta|e|\right. \text {. }
$$

One can see that at $V>0$ a single-electron resonant transmission dominates the current formation only, if $|e| V$ does not exceed the effective energy gap

$$
\Delta E_{L}^{(\mathrm{eff})}=\Delta E_{L}(0)+U \Phi(11 ; 11) / \eta .
$$

Note that this gap is defined by the position of the electrochemical potential relative to the single-electron energy (the gap $\Delta E_{L}(0) / \eta$ ) as well as by the Coulomb contribution, $U \Phi(11 ; 11)$. In the same manner one can derive the expressions for the critical voltages $V_{R}^{(1 \text { res })}$ and $V_{R}^{(2 \text { res })}$ related to the resonant electron transmission at negative values of $V$. Related expressions follow from Eqs. (52) and (53) if one replaces the quantities $\Delta E_{L}(0)$ and $\eta$ by the $\Delta E_{R}(0) \equiv \epsilon_{1}-E_{\mathrm{F}}$ and $1-\eta$, respectively. Thus, at low temperatures a single-electron transmission is efficient at $V<V_{L}^{(2 \text { res })}(V>0)$ or $|V|<V_{R}^{(2 \text { res })}(V<0)$. If $V(|V|)$ becomes comparable to $V_{L}^{(2 \text { res })}\left(V_{R}^{(2 \text { res })}\right)$ or exceeds it, the current 
is realized via channels where one or more excess electrons are already captured by the molecule before the steady current is formed [80]. At finite temperatures, due to thermally activated lead-molecule/molecule-lead electrontransfer processes the observation of the resonant transmission is smoothed. Nevertheless, the presence of two type of critical voltages associated with transmission through, respectively, the "empty" and singly occupied molecule, is physically undoubted. The derivation of respective expressions for $V_{L}^{(1 \text { res })}\left(V_{R}^{(1 \text { res })}\right)$ and $V_{L}^{(2 \text { res })}\left(V_{R}^{(2 \text { res })}\right)$ will be the subject of separate studies.

In the present paper we will concentrate on electron transmission processes along single-electron channels only. Note that a similar problem has been already discussed for the sequential mechanism of current formation in a short molecular chain $[63,64]$. However, here we consider another case for which the current proceeds through extended MOs. It supposes the presence of strong inter-site coupling $V_{m m^{\prime}}$. Moreover, we have to take into consideration a direct lead-lead transmission. The noted single-electron channel is characterized by the set of initial occupation numbers $\{N\}$ where all MOs $v$ are empty, i.e. $N_{v \sigma_{v}}=0$. If in the course of the charge transfer an electron leaves a lead and occupies the $\mu$ th MO, then the occupation number $N_{\mu \sigma_{\mu}}=0$ changes to $N_{\mu \sigma_{\mu}}^{\prime}=1-N_{\mu \sigma_{\mu}}=1$. After populating a molecular level the excess electron undergoes a relaxational transitions $\mu \rightleftharpoons \mu^{\prime}$ and forms an equilibrium distribution over all extended states of the molecule. Thus, the efficiency of the transmission depends on the relation between intra-molecular and molecule-lead kinetic processes. The situation is different if a direct lead-lead transmission along the same channel is considered. Now, the transmission proceeds in such a way that a singly occupied molecular state does not participate in intramolecular $\mu \rightleftharpoons \mu^{\prime}$ relaxation processes. It means that in Eq. (43) the condition $N_{v \sigma_{v}}=N_{v \sigma_{v}}^{\prime}=N_{v \sigma_{v}}^{\prime \prime}=0$ holds for all MOs except a certain MO $v=\mu$. For such a MO the relation between respective occupation numbers reads as $N_{\mu \sigma_{\mu}}^{\prime \prime}=1-N_{\mu \sigma_{\mu}}=1-N_{\mu \sigma_{\mu}}^{\prime}=1$. Bearing in mind the noted relations one may derive the concrete form of the nonlinear kinetic equations for all single-electron distribution functions (state populations).

The kinetic equations for the population of a given electronic state follows from expression (38). Let an electron with wave vector $\mathbf{k}$ and spin projection $\sigma_{\mathbf{k}}$ be in the conduction band of the left lead. Putting in Eq. (38) $j=L \mathbf{k}, \sigma_{j}=\sigma_{\mathbf{k}}$, then, one derives

$$
\begin{aligned}
\dot{P}_{L \mathbf{k} \sigma_{\mathbf{k}}}(t)= & -\frac{2 \pi}{\hbar} \sum_{\mu \sigma_{\mu}} \sum_{\lambda \alpha_{\mu}}\left|V_{L \mathbf{k} \lambda, \mu \alpha_{\mu}}\right|^{2} \delta_{\sigma_{\mathbf{k}}, \sigma_{\mu}}\left(1-\delta_{\sigma_{\mu}^{\prime}, \sigma_{\mu}}\right)\left[W\left(\varepsilon_{\lambda}^{(0)}\right) P_{L \mathbf{k} \sigma_{\mathbf{k}}}(t)\left(1-P_{\mu \sigma_{\mu}}(t)\right)-W\left(\varepsilon_{\mu \alpha_{\mu}}\right)\left(1-P_{L \mathbf{k} \sigma_{\mathbf{k}}}(t)\right) P_{\mu \sigma_{\mu}}(t)\right] \\
& \times L\left(E_{L \mathbf{k}}-\Delta \varepsilon_{\mu \alpha_{\mu}, \lambda}\right)\left(1-P_{\mu \sigma_{\mu}^{\prime}}(t)\right) \prod_{v \sigma_{v} \neq \mu \sigma_{\mu}, \sigma_{\mu}^{\prime}}\left(1-P_{v \sigma_{v}}(t)\right)-\frac{2 \pi}{\hbar} \sum_{\lambda \lambda^{\prime}} \sum_{\sigma_{\mathbf{k}} \sigma_{\mathbf{q}}}\left|T_{R \mathbf{q} \lambda^{\prime}, L \mathbf{k} \lambda}\right|^{2} \delta_{\sigma_{\mathbf{k}}, \sigma_{\mathbf{q}}}\left[W\left(\varepsilon_{\lambda}^{(0)}\right) P_{L \mathbf{k} \sigma_{\mathbf{k}}}(t)\right. \\
& \left.\times\left(1-P_{R \mathbf{q} \sigma_{\mathbf{q}}}(t)\right)-W\left(\varepsilon_{\lambda^{\prime}}^{(0)}\right) P_{L \mathbf{q} \sigma_{\mathbf{q}}}(t)\left(1-P_{L \mathbf{k} \sigma_{\mathbf{k}}}(t)\right)\right] L\left(E_{L \mathbf{k}}-E_{R \mathbf{q}}+\Delta \varepsilon_{\lambda, \lambda^{\prime}}^{(0)} \prod_{v \sigma_{v}}\left(1-P_{v \sigma_{v}}(t)\right) .\right.
\end{aligned}
$$

The matrix elements $V_{L \mathbf{k} \lambda, \mu \alpha_{\mu}}$ are defined by the expression (9) while we have

$$
T_{R \mathbf{q} \lambda^{\prime}, L \mathbf{k} \lambda}=\sum_{\mu \alpha_{\mu}} \frac{V_{L \mathbf{k} \lambda, \mu \alpha_{\mu}}^{*} V_{R \mathbf{q} \lambda^{\prime}, \mu \alpha_{\mu}}}{E_{L \mathbf{k}}-\mathscr{E}_{\mu \alpha_{\mu}}-\varepsilon_{\lambda}^{(0)}} \approx \sum_{\mu \alpha_{\mu}} \frac{V_{L \mathbf{k} \lambda, \mu \alpha_{\mu}}^{*} V_{R \mathbf{q} \lambda^{\prime}, \mu \alpha_{\mu}}}{E_{R \mathbf{q}}-\mathscr{E}_{\mu \alpha_{\mu}}-\varepsilon_{\lambda^{\prime}}^{(0)}} .
$$

The broadened $\delta$-functions (Lorentzian) $L\left(E_{L \mathbf{k}}-\Delta \varepsilon_{\mu \alpha_{\mu}, \lambda}\right)$ in Eq. (55) follow from Eq. (A.28) where one has to replace $\gamma_{\mu \alpha_{\mu}}$ by $\Gamma_{\mu \alpha_{\mu}}$, Eq. (20) while the Lorentzian $L\left(E_{L \mathbf{k}}-E_{R \mathbf{q}}+\Delta \varepsilon_{\lambda, \lambda^{\prime}}^{(0)}\right)$ is represented by Eq. (A.30).

The current is defined by the time-derivative of the integral lead population $N_{L}(t)$, (cf. Eqs. (11) and (12)). Therefore, to find $\dot{N}_{L}(t)$ one has to sum up both parts of Eq. (55) over $\mathbf{k}$ and $\sigma_{\mathbf{k}}$. Note, now, that $N_{L}(t)$ is a macroscopic quantity, and thus undergoes a small change during the electron transmission through the molecule, only. This means that $P_{L \mathbf{k} \sigma_{\mathbf{k}}}(t)$ deviates only a little from the equilibrium Fermi distribution (A.26). Therefore, we may set $P_{L \mathbf{k} \sigma_{\mathbf{k}}}(t) \simeq f_{L}\left(E_{L \mathbf{k}}\right)$ on the right-hand side of Eq. (55) (see the additional discussion in $[26,63,64]$ ). A similar approximation can also be introduced when one derives the kinetic equations for the populations $P_{\mu \sigma_{\mu}}(t)$. Since any magnetic field is absent we may set $P_{\mu \uparrow}(t)=P_{\mu \downarrow}(t) \equiv P_{\mu}(t)$, so that $\left(1-P_{\mu \uparrow}(t)\right)\left(1-P_{\mu \downarrow}(t)\right)=\left(1-P_{\mu}(t)\right)^{2}$.

Introducing the nonlinearity factors

$$
W_{0}(t)=\prod_{v \sigma_{v}}\left(1-P_{v \sigma_{v}}(t)\right)=\prod_{v}\left(1-P_{v}(t)\right)^{2}
$$

and

$$
W_{\mu}(t)=S_{\mu}(t) W_{0}(t),\left(S_{\mu}(t)=P_{\mu \sigma_{\mu}}(t) /\left(1-P_{\mu \sigma_{\mu}}(t)\right),\right.
$$

we rewrite the complete set of nonlinear kinetic equations describing the charge transmission along the single-electron channel. It follows 


$$
\begin{aligned}
& \dot{N}_{L}(t)=-\sum_{\mu}\left(K_{L \rightarrow \mu} W_{0}(t)-K_{\mu \rightarrow L} W_{\mu}(t)\right)-\left(Q_{L}-Q_{R}\right) W_{0}(t), \\
& \left.\dot{P}_{\mu}(t)\right)=-\sum_{r=L, R}\left(K_{\mu \rightarrow r} W_{\mu}(t)-K_{r \rightarrow \mu} W_{0}(t)\right)-\sum_{\mu^{\prime}}\left(K_{\mu \rightarrow \mu^{\prime}} W_{\mu}(t)-K_{\mu^{\prime} \rightarrow \mu} W_{\mu^{\prime}}(t)\right) \quad\left(\mu, \mu^{\prime}=1,2, \ldots, N\right), \\
& \dot{N}_{R}(t)=-\sum_{\mu}\left(K_{R \rightarrow \mu} W_{0}(t)-K_{\mu \rightarrow R} W_{\mu}(t)\right)-\left(Q_{R}-Q_{L}\right) W_{0}(t),
\end{aligned}
$$

where the single-electron rate constants $(r=L, R)$,

$$
K_{r \rightarrow \mu}=2 \sum_{\mathbf{k}} K_{r \mathbf{k} \rightarrow \mu}, K_{\mu \rightarrow r}=2 \sum_{\mathbf{k}} K_{\mu \rightarrow r \mathbf{k}},
$$

are defined through respective lead-molecule and molecule-lead transfer rates. The $K_{r \mathbf{k}} \vec{c}^{\mu}{ }^{\mu}$ are given in Eqs. (A.24) and (A.32). The $K_{\mu \rightarrow r \mathbf{k}}$ follow from the Eqs. (A.24) and (A.32) if one replaces $f_{L}\left(E_{L \mathbf{k}}\right) W\left(\varepsilon_{\lambda}^{(0)}\right)$ by $W\left(\varepsilon_{\mu \alpha_{\mu}}\right)$. The quantity

$$
Q_{L(R)}=2 \sum_{\mathbf{k q}} K_{L \mathbf{k} \rightarrow R \mathbf{q}(R \mathbf{k} \rightarrow L \mathbf{q})}
$$

denotes an integral transfer rate which characterizes the direct lead-lead electron transmission (see Eqs. (A.29) and (56)).

Our aim is to derive an expression for the stationary interelectrode current. For such a situation the electronic population of the molecule does not changes during electron transmission, i.e. $\dot{P}_{\mu}(t)=0$. Accordingly, the set of nonlinear equations (59) essentially simplifies. In particular, the electronic populations can be derived from the set of linear inhomogeneous equations for the auxiliary quantities $S_{\mu}$, Eq. (58),

$$
\sum_{\mu^{\prime}}\left[\left(K_{\mu \rightarrow L}+K_{\mu \rightarrow R}+\sum_{v} K_{\mu \rightarrow v}\right) \delta_{\mu \mu^{\prime}}-K_{\mu^{\prime} \rightarrow \mu}\right] S_{\mu^{\prime}}=K_{L \rightarrow \mu}+K_{R \rightarrow \mu}
$$

Note that the $S_{\mu}$ and thus the populations $P_{\mu}$ are exclusively defined by inelastic transfer processes within the LMRsystem. Solving the set of Eq. (62) and using the definition of $S_{\mu}$, we find the stationary electron populations $P_{\mu}$ together with the factors $W_{0}$ and $W_{\mu}$. The latter determine $\dot{N}_{L}$ according to Eq. (62). The expression for the current, Eq. (11) reduces to a sum of two contributions

$$
I=I_{\mathrm{dir}}+I_{\mathrm{int}},
$$

where the first contribution,

$$
I_{\mathrm{dir}}=-e W_{0}\left(Q_{L}-Q_{R}\right)
$$

refers to a direct lead-lead electron transmission while the second one,

$$
I_{\text {int }}=-e W_{0} \sum_{\mu}\left(K_{L \rightarrow \mu}-K_{\mu \rightarrow L} S_{\mu}\right)
$$

reflects electron transmission through intermediate LMR electronic states accompanied by a population of the extended molecular levels by the extra electron (the transferred electron). If the rate constants $K_{\mu \rightarrow v}$ characterizing the transitions between extended molecular states, are smaller than the electron-transfer rates $K_{\mu \rightarrow L(R)}$, the set of Eq. (62) has the simple solution, $S_{\mu}=\left(K_{L \rightarrow \mu}+K_{R \rightarrow \mu}\right) /\left(K_{\mu \rightarrow L}+K_{\mu \rightarrow R}\right)$ and thus the stationary populations are given by the expression

$$
P_{\mu}=\frac{K_{L \rightarrow \mu}+K_{R \rightarrow \mu}}{K_{L \rightarrow \mu}+K_{R \rightarrow \mu}+K_{\mu \rightarrow L}+K_{\mu \rightarrow R}} .
$$

\section{Discussion of the results}

The derivation of the set of nonlinear kinetic equations (38) for the reduced single-electron distribution functions at the presence of a Coulomb interaction between the transferred electrons has to be considered as the main result of the paper. It has been found that the related transfer rates, Eq. (36) include contributions from different transmission channels. Each of them refers to a definite number of excess electrons captured earlier by the molecule in the course of charge transfer. Main emphasis has been put on the single-electron transmission channel. It may participate in the charge transfer process if the molecule does not contain any excess electron. The probability of electron transfer through the single-electron channel is defined by the statistical factor $W_{0}$, Eq. (57). If the population of any electronic 
state $P_{\mu}$ increases, then, $W_{0}$ decreases and the current through the molecule may vanish. In the subsequent sections we will consider this problem in more detail.

\subsection{The direct lead-lead contribution to the single-electron channel of the current}

Using the definitions (A.29), (56) and (61), we may introduce the spectral density operator $\Gamma^{(r)}(r=L, R)$ and Green's operator $G(E)$ via their respective matrix elements

$$
\Gamma_{\mu \alpha_{\mu} \nu \beta_{v}}^{(r)}(E)=2 \pi \sum_{\mathbf{k}} V_{r \mathbf{k} \lambda, \nu \beta_{v}} V_{r \mathbf{k} \lambda, \mu \alpha_{\mu}}^{*} \delta\left(E-E_{L \mathbf{k}}\right)
$$

and

$$
G_{\mu \alpha_{\mu} \nu \beta_{v}}(E)=\frac{\delta_{\mu \alpha_{\mu}, \nu \beta_{v}}}{E-\mathscr{E}_{\mu \alpha_{\mu}}} .
$$

[Form (68) follows also from a general expression (44) at $E=E_{L \mathbf{k}}+\varepsilon_{\lambda}^{(0)}$, i.e. at $N_{L \mathbf{k} \sigma_{\mathbf{k}}}=1, N_{L \mathbf{k} \sigma_{\mathbf{k}}}^{\prime \prime}=0, N_{\mu \sigma_{\mu}}=0$, $N_{\mu \sigma_{\mu}}^{\prime \prime}=1, N_{v \sigma_{v}}=N_{\mu \sigma_{\mu}}^{\prime \prime}=0$.] Then, we may rewrite Eq. (64) to get

$$
I_{\mathrm{dir}}=W_{0} I_{\mathrm{dir}}^{(\mathrm{q}-\mathrm{e})}
$$

where we introduced the quasi-elastic current

$$
\begin{aligned}
I_{\mathrm{dir}}^{(\mathrm{q}-\mathrm{e})}= & -\frac{2 e}{h} \sum_{\lambda \lambda^{\prime}} \int_{-\infty}^{\infty} \int_{-\infty}^{\infty} \mathrm{d} E \mathrm{~d} E^{\prime} L\left(E-E^{\prime}\right) T_{\lambda \lambda^{\prime}}\left(E^{\prime}, E ; V\right)\left[W\left(\varepsilon_{\lambda}^{(0)}\right) f_{L}\left(E-\varepsilon_{\lambda}^{(0)}\right)\left(1-f_{R}\left(E^{\prime}-\varepsilon_{\lambda^{\prime}}^{(0)}-e V\right)\right)\right. \\
& \left.-W\left(\varepsilon_{\lambda^{\prime}}^{(0)}\right)\left(1-f_{L}\left(E-\varepsilon_{\lambda}^{(0)}\right)\right) f_{R}\left(E^{\prime}-\varepsilon_{\lambda^{\prime}}^{(0)}-e V\right)\right] .
\end{aligned}
$$

This current is formed by single-electron transmission if any electron-electron correlation in the course of electron motion has completely be ignored. The conducting properties of the molecule are covered by the transmission function

$$
T_{\lambda \lambda^{\prime}}\left(E^{\prime}, E ; V\right)=\operatorname{Tr}\left(\Gamma^{(R)}\left(E^{\prime}-\varepsilon_{\lambda^{\prime}}^{(0)}\right) G(E) \Gamma^{(L)}\left(E-\varepsilon_{\lambda}^{(0)}\right) G^{+}(E)\right) .
$$

The expression for the elastic current, $I_{\text {dir }}^{(\text {el) }}$, follows from Eq. (71) valid for a quasi-elastic current if $\varepsilon_{\lambda^{\prime}}^{(0)}=\varepsilon_{\lambda}^{(0)}$ and if $L\left(E-E^{\prime}\right)=\delta\left(E-E^{\prime}\right)$. As long as the $I-V$ characteristics are measured at room temperatures or below the estimate of the current can be performed by replacing the Fermi function $f_{s}(\epsilon)$ by the unit-step function $\theta\left(E_{\mathrm{F}}-\epsilon\right)$ [55]. It yields

$$
I_{\mathrm{dir}}^{(\mathrm{q}-\mathrm{e})} \simeq I_{\mathrm{dir}}^{(\mathrm{el})}=\sum_{\lambda} W\left(\varepsilon_{\lambda}^{(0)}\right) I_{\lambda}^{(\mathrm{el})} .
$$

The partial elastic current through the molecule (note $e<0$ ),

$$
I_{\lambda}^{(\mathrm{el})}=\frac{2 e}{h} \int_{E_{\mathrm{F}}}^{E_{\mathrm{F}}+e V} \mathrm{~d} E T_{\lambda \lambda}(E, E ; V),
$$

displays the contribution from the transmission along the single-electron transmission channel when the molecule (without an extra electron) is in the $\lambda$ th vibrational state. At low temperature, the elastic current is formed by the molecule which is in its vibrational state-ground. Putting $\varepsilon_{0}^{(0)}=0$ we arrive at the standard expression for the elastic interelectrode current [60-62] so that

$$
I_{\mathrm{dir}}^{(\mathrm{q}-\mathrm{e})} \approx I^{(\mathrm{el})}=\frac{2 e}{h} \int_{E_{\mathrm{F}}}^{E_{\mathrm{F}}+e V} \mathrm{~d} E T(E, V),
$$

where

$$
T(E, V) \equiv \operatorname{Tr}\left(\Gamma^{(R)}(E) G(E) \Gamma^{(L)}(E) G^{+}(E)\right) .
$$

\subsection{The inelastic contribution to the single-electron channel of the current}

This contribution to the overall current proceeds through intermediate states populated by the transferred electron. We will restrict the considerations to the case where the lead-molecule (molecule-lead) transitions are much faster than relaxation processes between the molecular levels $\mu$ and $\mu^{\prime}$. Therefore, Eq. (66) can be used and, thus, the expression for the inelastic current, Eq. (65) takes the following form: 


$$
I_{\text {int }}=-e \sum_{\mu} W_{0 \mu} \frac{K_{L \rightarrow \mu} K_{\mu \rightarrow R}-K_{R \rightarrow \mu} K_{\mu \rightarrow L}}{K_{L \rightarrow \mu}+K_{R \rightarrow \mu}+K_{\mu \rightarrow L}+K_{\mu \rightarrow R}}
$$

with

$$
W_{0 \mu}=\left(1-P_{\mu}\right) \prod_{v \neq \mu}\left(1-P_{v}\right)^{2} .
$$

According to the definitions (60) and (A.24) and after replacing the Lorentzian by a $\delta$-function the lead-molecule and molecule-lead transfer rates read

$$
\begin{aligned}
& K_{\mu \rightarrow L(R)}=\left(Z_{0} / Z_{\mu}\right) \mathrm{e}^{\Delta E_{\mu L(R)}(V) / k_{\mathrm{B}} T} K_{L(R) \rightarrow \mu}, \\
& K_{L \rightarrow \mu}=\frac{2}{\hbar} \sum_{\lambda \alpha_{\mu}} \Gamma_{\lambda \mu \alpha_{\mu} \lambda \mu \alpha_{\mu}}^{(L)}\left(\varepsilon_{\mu \alpha_{\mu}}-\varepsilon_{\lambda}^{(0)}\right) W\left(\varepsilon_{\lambda}^{(0)}\right) f_{L}\left(\varepsilon_{\mu \alpha_{\mu}}-\varepsilon_{\lambda}^{(0)}\right), \\
& K_{R \rightarrow \mu}=\frac{2}{\hbar} \sum_{\lambda \alpha_{\mu}} \Gamma_{\lambda \mu \alpha_{\mu} \lambda \mu \alpha_{\mu}}^{(R)}\left(\varepsilon_{\mu \alpha_{\mu}}-\varepsilon_{\lambda}^{(0)}\right) W\left(\varepsilon_{\lambda}^{(0)}\right) f_{R}\left(\varepsilon_{\mu \alpha_{\mu}}-\varepsilon_{\lambda}^{(0)}-e V\right) .
\end{aligned}
$$

The partition functions $Z_{0}$ and $Z_{\mu}$ are defined in Eqs. (A.6) and (A.27), whereas $\Delta E_{\mu L}(V)$ and $\Delta E_{\mu R}(V)$ are the energy differences between the $\mu$ th $\mathrm{MO}$ and the Fermi level of the left and right lead, respectively (Eq. (88)).
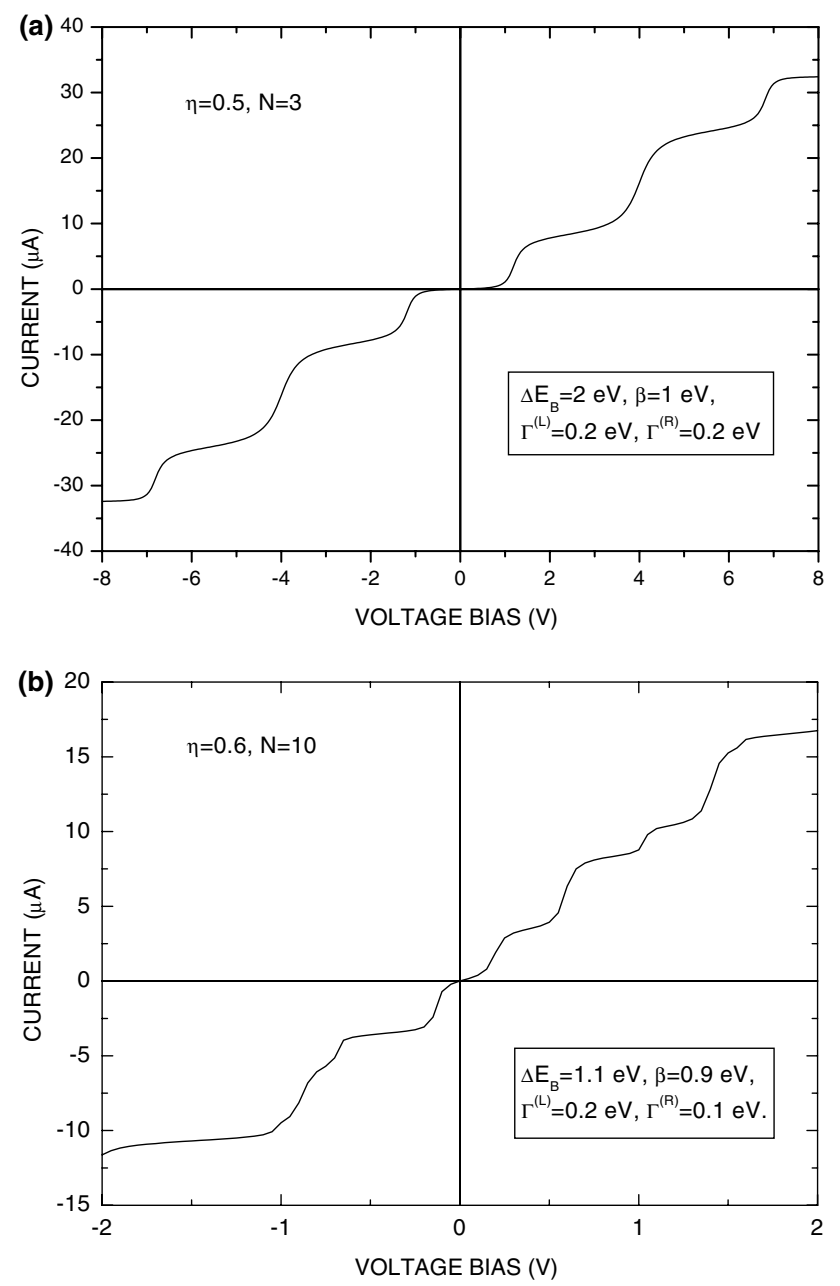

Fig. 4. Direct lead-lead single-electron current through a linear 3-site (a) and 10-site molecule (b). The transmission occurs through 3 or 10 extended MOs, respectively. Inelastic processes are not taken into consideration. The calculations are based on Eqs. (80) and (86) at $W_{0}=1$. 


\subsection{Interplay between the elastic and inelastic transmission}

We compare the influence of the different contribution to the overall current (63) in using the same parameters that define the elastic and inelastic transmission. To this end we consider a vibration-less electron transmission for which the elastic lead-lead current is given by Eqs. (69) and (74). It is possible to show (see also [79]) that for strong inter-site couplings $V_{m m \pm 1}$ the transmission function (75) reduces to

$$
T(E, V)=\Gamma^{(L)} \Gamma^{(R)}\left|\sum_{\mu=1}^{N} \frac{u_{\mu}(1) u_{\mu}^{*}(N)}{E-\mathscr{E}_{\mu}+\mathrm{i} 0^{+}}\right|^{2} .
$$

The complex energy of the $\mu$ th molecular state,

$$
\mathscr{E}_{\mu}=\varepsilon_{\mu}+\eta e V-\mathrm{i} \gamma_{\mu}
$$

includes a voltage induced shift which is characterized by voltage division factor (cf. Fig. 1)

$$
\eta=\left[1+\left(\delta_{L}-\delta_{R}\right) / \delta\right] / 2
$$

while

$$
\gamma_{\mu}=\left[\left|u_{\mu}(1)\right|^{2} \Gamma^{(L)}+\left|u_{\mu}(N)\right|^{2} \Gamma^{(R)}\right] / 2
$$

is the broadening of the $\mu$ th level. It is caused by the lead-molecule interactions $\left(\Gamma^{(L(R))}\right.$ is the level broadening related to left (right) terminal unit of the molecule). For a sake of clarity we consider a molecule with a regular arrangement of
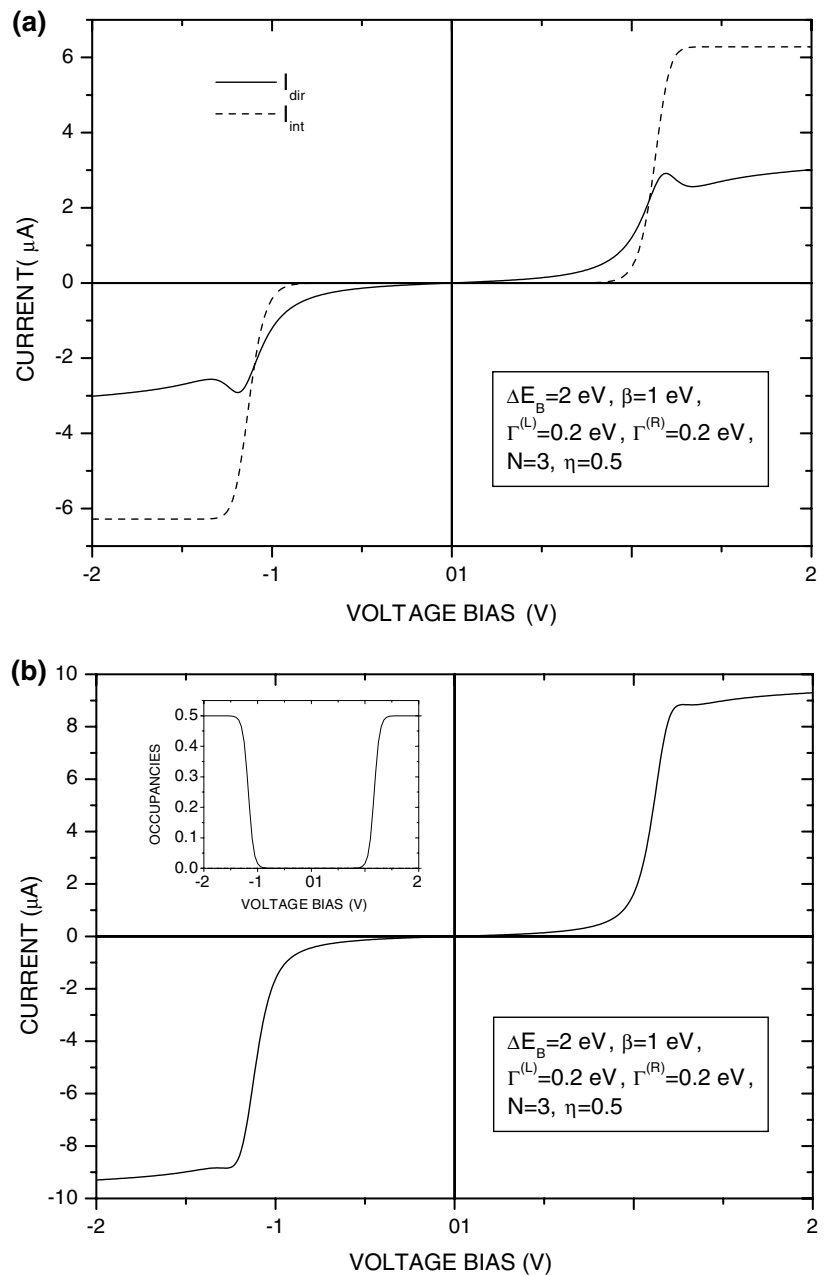

Fig. 5. Direct lead-lead current $I_{\text {dir }}$ and the inelastic current $I_{\text {int }}$ formed at the presence of a population of an intermediate state (a). Total current through the molecule, $I=I_{\mathrm{dir}}+I_{\mathrm{int}}$, and the state population $P_{\mu=1}$ of the lowest molecular level (b). Symmetric case, calculations according to Eqs. (76), (80), (86), (57) and (77). 
$N$ identical sites. It means that in the absence of a voltage bias $V$ the site energies $E_{m}$ coincide, $E_{1}=E_{2}=\cdots=$ $E_{N} \equiv E_{B}$. The same should hold for the inter-site couplings, $V_{m m \pm 1} \equiv \beta$. Therefore, we may write

$$
\begin{aligned}
& \varepsilon_{\mu}=E_{B}-2 \beta \cos \frac{\pi \mu}{N+1}, \\
& u_{\mu}(n)=\sqrt{\frac{2}{N+1}} \sin \frac{\pi \mu n}{N+1} \quad(\mu=1,2, \ldots, N) .
\end{aligned}
$$

It follows from Eqs. (80) and (81) that

$$
T(E, V)=T(E-\eta e V) \equiv T(x),
$$

and, thus, we obtain the following expression for the direct lead-lead current

$$
I_{\mathrm{dir}}=W_{0} I_{0} \int_{E_{\mathrm{F}}-(1-\eta)|e| V}^{E_{\mathrm{F}}+\eta|e| V} \mathrm{~d} x T(x),\left(I_{0} \equiv 2|e| / h\right) .
$$

Here, the transmission function $T(x)$ does not depend on the voltage bias $V$. The shape of the $I-V$ characteristics is caused by the integration limits as well as by the factor $W_{0}$. Note that the expression for the lead-lead current agrees with the standard expression for the elastic current provided that the electronic populations become small $\left(P_{\mu} \ll 1\right)$ and thus $W_{0} \simeq 1$. If it is not the case, then, due to inelastic processes the molecule is populated by the transferred electrons. As a result, $W_{0}$ modifies the transmission through a single-electronic channel just reducing the current through this channel.
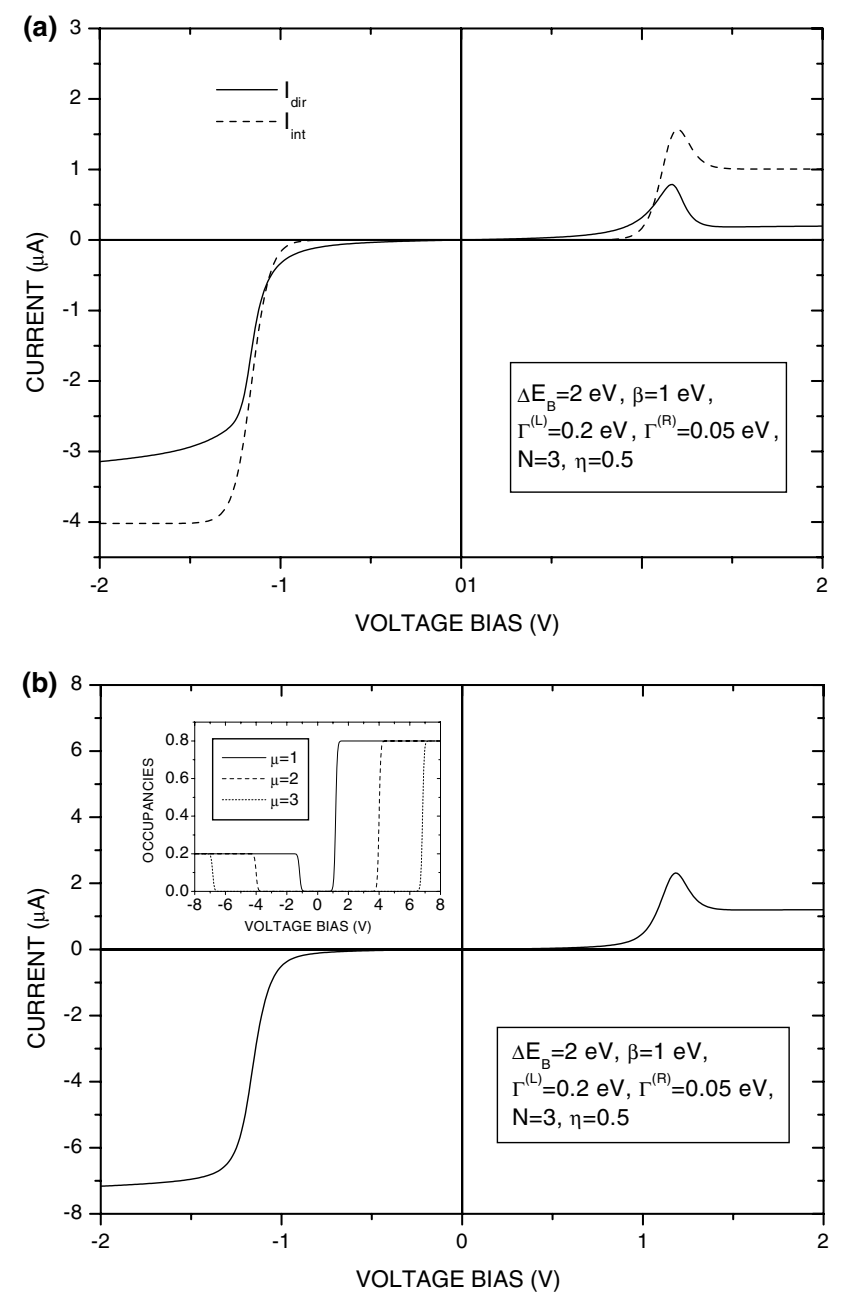

Fig. 6. Direct lead-lead and inelastic currents (a). Total current and state populations of the molecule (b). Asymmetric case, calculations are based on Eqs. (76), (80), (86), (57) and (77). The asymmetry is related to the difference between the left lead-molecule and the right lead-molecule inelastic transfer processes. 
Assuming the same conditions as beforehand, the expression (76) for an inelastic current is reduced to

$$
I_{\text {int }}=I_{0} \pi \Gamma^{(L)} \Gamma^{(R)} \sum_{\mu} W_{0 \mu}\left(\left|u_{\mu}(1) u_{\mu}(N)\right|^{2} / \gamma_{\mu}\right)\left(\frac{1}{\mathrm{e}^{\Delta E_{\mu L} / k_{\mathrm{B}} T}+1}-\frac{1}{\mathrm{e}^{\Delta E_{\mu R} / k_{\mathrm{B}} T}+1}\right)
$$

where energy gaps read (note $\Delta \varepsilon_{\mu}=\varepsilon_{\mu}-E_{\mathrm{F}}$ )

$$
\Delta E_{\mu L}(V)=\Delta \varepsilon_{\mu}-\eta|e| V, \quad \Delta E_{\mu R}(V)=\Delta \varepsilon_{\mu}+(1-\eta)|e| V .
$$

Fig. 4 displays the typical steplike $I-V$ characteristics of a molecule for the case where the current is formed by a direct single-electron lead-lead transmission. The MOs are not populated by the transferred electrons and the lead-lead transmission proceeds in an elastic manner. The number of steps in the $I-V$ characteristics which might become visible is given by the number of levels $N$. If a model with a single localized MO per site is taken then $N$ coincides with the number of sites (as it is the case for Fig. 4). The smooth transition from one step to subsequent step is caused by the level broadening $\Gamma^{(L)}$ and $\Gamma^{(R)}$. An asymmetry of the $I-V$-curves with respect to $V>0$ and $V<0$ appears if the voltage division factor $\eta$ differs from 0.5 (compare Fig. 4(a) and (b)).

The shape of the $I-V$-curves essentially changes if an inelastic pathway contributes. Now the transferred electron undergoes relaxational transitions in the molecule. The MOs are populated by the excess electron and the level populations $P_{\mu}$ differ from zero. This leads to a change of the factors $W_{0}$, Eq. (57) and $W_{0 \mu}$, Eq. (77), which are responsible for a change of the elastic and inelastic component of the current. An expression for both components can be found in Eqs. (86) and (87). [However, it is necessary to remind on the fact that Eqs. (86) and (87) are only correct if the inequalities (23) and (24) are fulfilled together with $V<V_{L}^{(2 \mathrm{res})}$ or $|V|<V_{R}^{(2 \mathrm{res})}$ (cf. definition (53)). Just the validity of the latter condition guarantees that the Coulomb interaction does not change the single-electron rate constants given in Eqs.
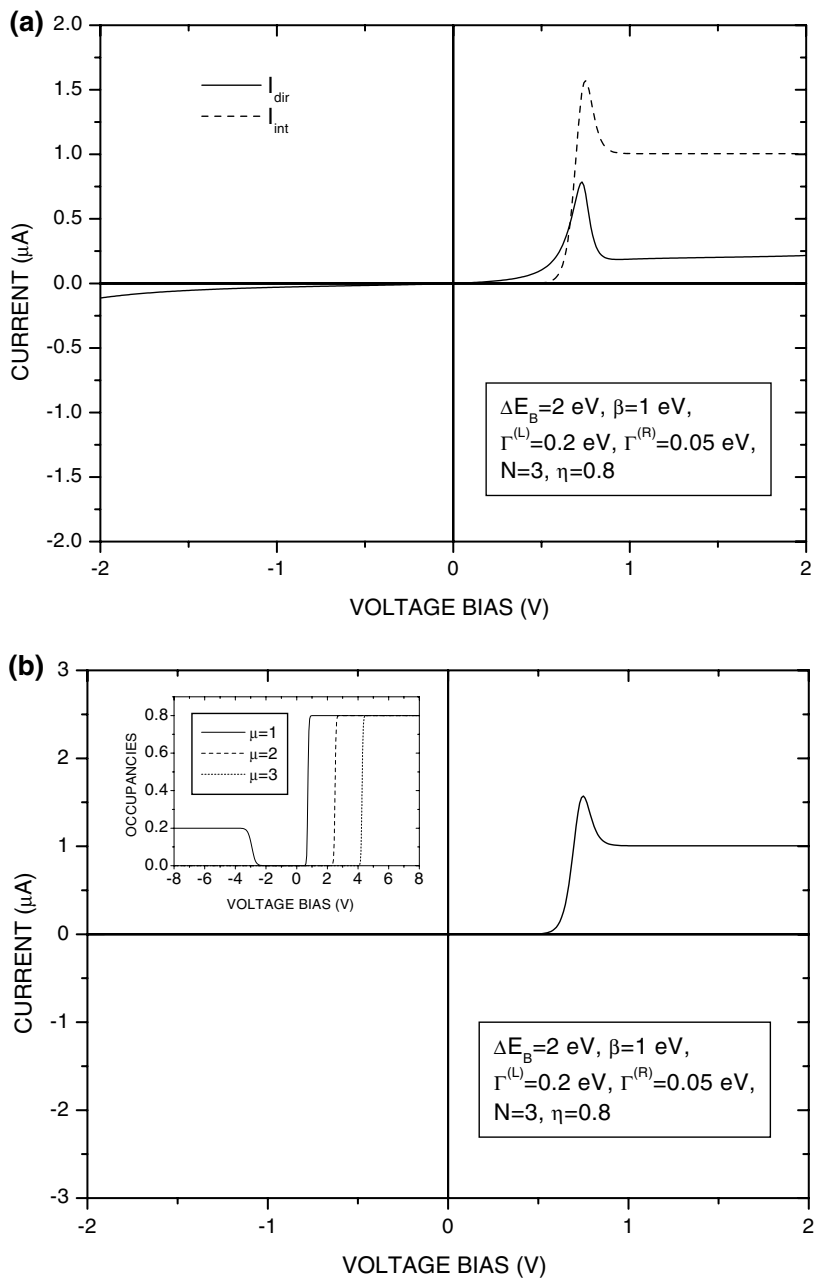

Fig. 7. Asymmetric current-voltage characteristics of a molecule. Calculations are based on Eqs. (76), (80), (86), (57) and (77). The asymmetry is related to the difference between left lead-molecule and the right lead-molecule inelastic transfer processes as well as to the voltage division factor $\eta$. 
(A.24) and (A.29).] To become more concrete let us consider a 3 -site molecule and let us take $\beta=2 \mathrm{eV}, U=3 \mathrm{eV}$. Now, the inequalities (23) and (24) are valid for an arbitrary voltage bias. Although the $I-V$ characteristics of the 3 -site molecule includes three steps (cf. Fig. 4(a)) the inequality $V<V_{L}^{(\text {2res })}\left(|V|<V_{R}^{(2 \text { res })}\right.$ ) restricts the correct computation of the current to a certain voltage range. For an energy gap $\Delta E_{B}=E_{B}-E_{\mathrm{F}}=2 \mathrm{eV}$, this range is given -2 to $2 \mathrm{~V}$, where only the first step of the complete $I-V$ curve is included. Just this step is shown in Figs. 5-7. If the broadening $\Gamma^{(L)}$ and $\Gamma^{(R)}$ coincide and if the voltage division factor $\eta$ equals 0.5 the $I-V$ characteristics becomes symmetric (cf. Fig. 5). Note, that the direct lead-lead contribution to the current shows a non-monotonic behavior in the region 1 to $1.5 \mathrm{~V}$ and in the region -1 to $-1.5 \mathrm{~V}$ (see also Fig. 5(a)). Such behavior of the $I-V$ curves is caused by the increase of the lowest level population $P_{1}$ from zero to 0.5 (cf. the insert in Fig. 5(b)). Correspondingly, the quantities $W_{0}$ and $W_{0 \mu}$ also changes. It is worth mentioning that for $|V|>1.2 \mathrm{~V}$, just originated by $W_{0}$ and $W_{0 \mu}$, the inelastic current, $I_{\text {int }}$, exceeds the modified elastic current, $I_{\mathrm{dir}}$. Consequently the total current $I$, Eq. (64) is formed by an elastic and inelastic contribution (see Fig. $5(\mathrm{~b})$ ). If the broadening $\Gamma^{(L)}$ and $\Gamma^{(R)}$ strongly differ a pronounced asymmetry in voltage dependence of $I_{\mathrm{dir}}, I_{\mathrm{int}}$, and of the total current $I$ appears (compare Fig. 6(a) and (b)). The asymmetry in the I-V curves (the rectification effect) is enforced if the voltage division factor $\eta$ deviates from 0.5 . One may highlight this result by comparing the regions $V>0$ and $V<0$ in Figs. 6 and 7.

The influence of the factor $\eta$ on the rectification properties of a single molecule is determined by the concrete form of the voltage drop in the interelectrode region. This problem has been discussed in different papers (see, e.g., $[55,60,61,74,79,82,83])$. Therefore, we will concentrate on a different rectification effect which is related to the inelastic transfer. To this end let us consider electron transmission through a molecule which is symmetrically disposed the two lead surfaces so that $\eta=0.5$. In this case the inequality $\Gamma^{(L)} \neq \Gamma^{(R)}$ becomes valid if the contact of the molecule with the left lead differs from that with the right lead (for instance, due to different types of terminal groups of the molecule). The difference between $\Gamma^{(L)}$ and $\Gamma^{(R)}$ results in a non-symmetric electron transfer and the left lead $\rightarrow$ right lead transmission differs from the right lead $\rightarrow$ left lead one. This results in different values of the stationary population $P_{1}$ for $V>0$ and for $V<0$ (cf. insert of Figs. 6(b) and 7(b)). Since the voltage dependence of $W_{0}$ and $W_{0 \mu}$ follows from that of $P_{1}$, an asymmetric dependence of $I_{\mathrm{dir}}$ and $I_{\text {int }}$ on the applied voltage is also caused by that of $P_{1}$. [As in the previously

(a)

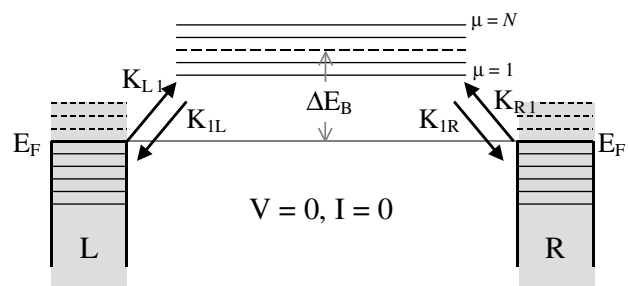

(b)

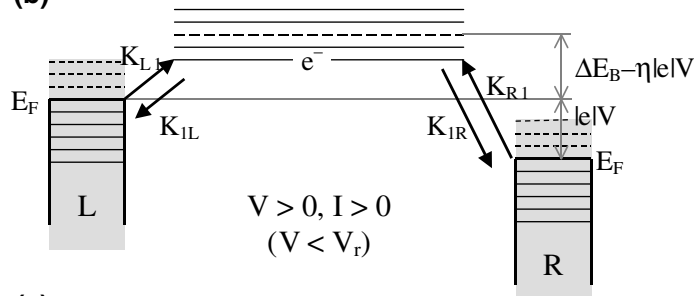

(c)

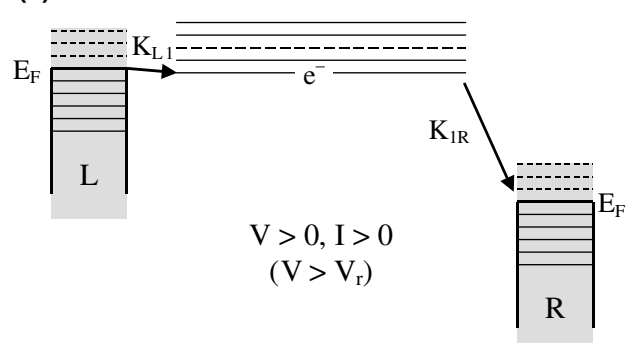

Fig. 8. Kinetics of an inelastic process responsible for the appearance of non-zero steady state populations $P_{\mu}$. If $V<V_{r}$ the populations are small and thus no strong influence of the inelastic transfer process on the elastic one appears. For $V>V_{r}$ the populations are defined by the relation between the transfer rates of the incoming and of the outgoing charges. As an example, the inelastic transmission through the lowest energy level $\mu=1$ is shown. It is defined by the transfer rates $K_{L 1}$ and $K_{1 R}$, respectively. 
discussed symmetric case shown in Fig. 5(b), the populations $P_{2}$ and $P_{3}$ equal zero in the voltage range -2 to $2 \mathrm{~V}$. Therefore, $P_{2}$ and $P_{3}$ do not influence $W_{0}$ and $W_{0 \mu}$. Thus, a kinetic rectification effect appears resulting from different populations of the molecule by the transferred electron whether the left-right or the right-left inelastic transmission is considered. The formation of a non-symmetric stationary population $P_{1}$ caused by non-elastic transfer processes is illustrated in Fig. 8. Let $V_{r} \equiv V_{L}^{(1 \text { res) }}$ (cf. Eq. (52)) be the characteristic voltage at which resonance transmission appears via the lowest molecular level $\mu=1$. This voltage is defined by the condition $\Delta E_{1 L}(V)=0$ (at $V>0$ ) and $\Delta E_{1 R}(V)=0$ (at $V<0$, for the energy differences see Eq. (88)). If the voltage division factor $\eta$ equals 0.5 , then $V_{r}$ becomes independent on the voltage sign and equals $0.6 \mathrm{~V}$. [To estimate $V_{r}$ we took the parameters $\Delta E_{B}, \beta$ and $N$ identical to those used in Fig. 6.] Let us consider an inelastic transmission process at $V>0$. In the region $V<V_{r}$, the lead-molecule transfer rates $K_{L \rightarrow 1}$ and $K_{R \rightarrow 1}$ are much smaller than the respective lead-molecule transfer rates $K_{1 \rightarrow L}$ and $K_{1 \rightarrow R}$. Therefore, in accordance with Eq. (66) the population $P_{1}$ remains small. This yields $W_{0} \simeq 1, W_{0 \mu} \simeq 1$ (remember that in the region -2 to $2 \mathrm{~V}$ one obtains $P_{2} \simeq 0, P_{3} \simeq 0$ ). Thus, in the region $V<V_{r}$ any pronounced modification of both current components by $W_{0}$ and $W_{0 \mu}$ is absent. The situation changes in the vicinity $V \sim V_{r}$ where $P_{1}$ starts to increase. If $V>V_{r}$ and if the relations $K_{L \rightarrow 1} \gg K_{1 \rightarrow L}, K_{R \rightarrow 1} \ll K_{R \rightarrow 1}$ are fulfilled, the inelastic current is determined by the direct leftright electron transmission through an intermediate state associated with the population of the MO $\mu=1$ (cf. scheme (c) in Fig. 8). In the course of the directed left-right electron transfer the population of the intermediate state saturates at $P_{1} \simeq \Gamma^{(L)} /\left(\Gamma^{(L)}+\Gamma^{(R)}\right)=0.8$. If $V<0$ and if a direct right-left electron transfer takes place the saturated value is given by $P_{1} \simeq \Gamma^{(R)} /\left(\Gamma^{(L)}+\Gamma^{(R)}\right)=0.2$. One may notice the difference between the populations for $V>0$ and for $V<0$ (see also the insert of Fig. 6(b)). Therefore, $W_{0}$ and $W_{0 \mu}$ also changes considerably for $V>0$ and for $V<0$. Note again, that just this fact is responsible for the occurrence of non-symmetric $I-V$ characteristics of the single-electron current and thus for the observation of kinetic rectification effect.

\section{Conclusion}

In present paper we considered some general theoretical problems related to single-electron elastic and inelastic transmission through a molecular wire. To remain sufficient simple the wire has been represented by an $N$-site tight-binding model leading to $N$ delocalized MOs. If embedded in between two leads the MOs participate in the electron transmission leading to a net current which is caused by two different electron transfer routes between the leads. The first route is originated by an electron transfer process where the MOs are not populated by the transferred electron even if resonance conditions are fulfilled for the molecular levels. This route is responsible for formation of an elastic inter-electrode current. The other route is related to a two-step transmission where the transferred electron leaves the lead and undergoes relaxation in the wire including population of the MOs. Afterward the electron is transferred to another lead. This a transmission process forms the inelastic inter-electrode current.

It is usually assumed that in a left lead, molecule, right lead (LMR) system the current is formed either by an elastic or by an inelastic transmission process. Generally, this is not the case and both type of transmission may be simultaneously contribute to the current. A proper description of such a situation has been achieved by using the density matrix technique. The derived nonlinear kinetic equations for reduced single-electron distribution, Eq. (38) describe the elastic as well as the inelastic electron transfer. The kinetic equations are valid for different situations including those where the Coulomb interaction between the transferred electrons strongly affects the electron transmission through the molecule. It is important to note that in the framework of the unified description the elastic as well as the inelastic component of the current are expressed by a single set of parameters. This allows to correctly compare the contribution of both current components to the total inter-electrode current. We consider the derivation of the nonlinear kinetic equations (38) and the respective transfer rates (36) as the main result of our studies.

The unified description shows that the Coulomb interaction is responsible for the formation of the specific electrontransfer channels associated with the number of extra electrons captured by the molecule in the course of charge transmission. Among these channels the single-electron channel is of particular importance. This channel governs the transmission through an "empty" molecule, i.e. the molecule which does not contain an extra electron except the only one just being transferred. Therefore, the formation of the current through the single-electron channel proceeds in the absence of Coulomb interaction. However, the probability of elastic transmission through this channel is not equal unity but is given by the factor $W_{0}$, Eq. (57). It means that the direct lead-lead current $I_{\mathrm{dir}}$ is defined as a modified completely elastic current, Eq. (86). Moreover, the inelastic current, Eq. (87) is modified by the factor $W_{0 \mu}$, Eq. (77). The appearance of $W_{0}$ and $W_{0 \mu}$ is related to the fact that in the course of electron transmission the "empty" molecule is kinetically populated by the transferred electron. Accordingly, each molecular state population $P_{\mu}$, Eq. (66) is determined by the inelastic transfer processes. Our studies show that a completely elastic lead-lead current only appears at an negligible population of the molecule by the transferred electron (i.e. if $P_{\mu} \ll 1$ is valid for all 
molecular levels $\mu$ ). As one example for such a situation we refer to the superexchange lead-lead electron transmission. Another example is given by an asymmetric electron transmission where the broadenings $\Gamma^{(L)}$ and $\Gamma^{(R)}$ strongly differ one from another. Here, a small population of the molecule appears following from the directed inelastic transfer process (cf. scheme (c) in Fig. 8 and discussion to Figs. 6 and 7).

The described interrelation between the elastic and inelastic component of the total current has to be considered as further important result of the present paper. This conclusion also underlines that both components of the current give a comparable contribution to the total current (see Figs. 5-7). Although this result has been obtained via the analysis of low-temperature vibration-less electron transmission through the single-electron channel only, it reflects a general physical situation. Inelastic transfer processes lead to a population of the molecule by the transferred electrons. Accordingly, the direct lead-lead transmission proceeds via the transfer channels formed by extra electrons captured in the course of inelastic transfer. The number of captured electrons as well as the character of electron-transfer channels depends on relation between dynamic and relaxational characteristics of the LMR system (position of molecular levels with respect to the Fermi levels of the lead, Coulomb interaction between the extra (transferred) electrons, couplings to the lead, couplings to various vibrational modes and other). The opening of a particular channel is determined by the strength of the applied voltage. For instance, if the Coulomb interaction is taken in the Hubbard-form and if the inequalities (22) and (23) are fulfilled the single-electron channel may participate in charge transmission in the voltage region $V<V_{L}^{(2 r e s)}\left(|V|<V_{R}^{(2 \text { res })}\right)$. The corresponding lead-molecule/molecule-lead and lead-lead rate constants are defined by the expressions (86) and (87). Note, that the concrete expressions for these rate constants only follow from the general expression for the transfer rate (36), if one analyzes a vibration-less transmission. But, Eq. (36) contains contributions from the various electron-transfer processes as well. Moreover, it accounts for the broadening of the electron-vibrational levels of the molecule due to fast vibrational relaxation as well as due to the interaction with the macroscopic leads. [This is clearly demonstrated by Eqs. (47)-(50) specifying the lead-molecule transfer rates.] Therefore, the kinetic equations (38) are able to describe the formation of interelectrode current caused by various elastic and inelastic transmission channels including the thermally activated phonon-assisted channels.

The proposed unified description of electron-transfer processes refers to LMR system where the molecule or molecular wire positioned in between the leads is characterized by delocalized electronic states. The next step of our studies will the description of electron transfer through molecular structures where the electron transmission proceeds across localized electronic states.

\section{Acknowledgments}

One of us (E.G.P.) gratefully acknowledge generous support by the Alexander von Humboldt Foundation. P.H. also acknowledges the support by the DFG via the collaborative research grant SFB-486, project A-10.

\section{Appendix A. Kinetic equations for integral populations}

The main purpose of the appendix is to derive kinetic equations governing the single-electron state populations $P_{j \sigma_{j}}(t)$ at a situation where the molecule is coupled to the leads and the transmission occurs against the background of fast intra-term vibration relaxation. To this end we employ the density matrix approach for the LMR system additionally coupled to a thermal bath (heat bath) to include vibrational relaxation. A similar approach has already been used for the derivation of kinetic equations describing electron-vibrational dynamics in a donor-bridge-acceptor system $[73,77]$ as well as the LMR system studied here $[65,26]$. The kinetic equations are obtained from generalized master equations (GME) for the reduced density operator $\rho(t)$ governing the dynamics of an open quantum system like the considered LMR system [66-69].

To offer a sufficient clear description we especially consider (in Section A.1) the derivation of a master equation for the electron-vibrational level populations, Eq. (A.17). Moreover, it will be demonstrated in which way the fast vibrational relaxation justifies a reduced description via kinetic equations for integral level populations, Eq. (A.5). Sections A. 2 and A. 3 demonstrate how to account for the lead-molecule interaction in the kinetic equations (A.18) and how to compute single-electron rate constants (A.24) and (A.29).

\section{A.1. Kinetic equations for integral electronic state populations}

In the following we will study in detail the way wherein fast vibrational relaxation processes influence the kinetic processes within the molecule. Let us abbreviate the molecular electron-vibrational states $\left|\mu \sigma_{\mu} ; \chi_{\mu \alpha_{\mu}}\right\rangle$ by $|s \alpha\rangle$. [Note that 
in the occupation number representation $\left|\mu \sigma_{\mu} ; \chi_{\mu \alpha_{\mu}}\right\rangle=\left|1_{\mu \sigma_{\mu}}\right\rangle\left|\chi_{\mu \alpha_{\mu}}\right\rangle=a_{\mu \sigma_{\mu}}^{+}\left|0_{\mu \sigma_{\mu}}\right\rangle\left|\chi_{\mu \alpha_{\mu}}\right\rangle$.] Then, the GME in Markov approximation written for the matrix elements of the density operator $\rho(t)$,

$$
\rho_{s \alpha_{s} s^{\prime} \alpha_{s^{\prime}}}(t)=\left\langle s \alpha_{s}|\rho(t)| s^{\prime} \alpha_{s^{\prime}}\right\rangle,
$$

takes the following forms $[73,81]$ :

$$
\begin{aligned}
\dot{\rho}_{s \alpha s^{\prime} \beta}(t)= & -\mathrm{i} \omega_{s \alpha s^{\prime} \beta} \rho_{s \alpha s^{\prime} \beta}(t)-\frac{\mathrm{i}}{\hbar} \sum_{k \xi}\left(V_{s \alpha, k \xi} \rho_{k \xi^{\xi} s^{\prime} \beta}(t)-V_{k \xi_{, j} \beta} \rho_{s \alpha k \xi}(t)\right)-\left(1-\delta_{s, s^{\prime}}\right)(1 / 2)\left(\gamma_{s \alpha}+\gamma_{s^{\prime} \beta}\right) \rho_{s \alpha s^{\prime} \beta}(t) \\
& -\delta_{s, s^{\prime}} \delta_{\alpha, \beta} \sum_{\alpha^{\prime}}\left(w_{\alpha \rightarrow \alpha^{\prime}}^{(s)} \rho_{s \alpha . s \alpha}(t)-w_{\alpha^{\prime} \rightarrow \alpha}^{(s)} \rho_{s \alpha^{\prime} s \alpha^{\prime}}(t)\right) .
\end{aligned}
$$

The couplings matrix elements $V_{s \alpha, s^{\prime} \alpha^{\prime}}=\left\langle s \alpha\left|V_{\text {int }}\right| s^{\prime} \alpha^{\prime}\right\rangle$ are responsible for transitions between electron-vibrational states $|s \alpha\rangle$ and $\left|s^{\prime} \alpha^{\prime}\right\rangle$ related to different electronic levels $s$ and $s^{\prime}$ (spin quantum number is timely omitted). The quantity $\gamma_{s \alpha}=\tau_{s \alpha}^{-1}=\sum_{\alpha^{\prime}} w_{\alpha \rightarrow \alpha^{\prime}}^{(s)}$ defines the inverse life-time of the electron-vibrational state $|s \alpha\rangle$ and coincides with the broadening of the respective electron-vibrational level. To find the $\gamma_{s \alpha}$ one has to calculate the rate $w_{\alpha \rightarrow \alpha^{\prime}}^{(s)}$ which characterizes the relaxation between the vibrational states $|s \alpha\rangle$ and $\left|s \alpha^{\prime}\right\rangle$. It is possible to show that if the coupling to a heat bath is defined by $\Phi_{\alpha \alpha^{\prime}}^{(s)}$, Eq. (6) then $\left(s=\mu, \alpha=\alpha_{\mu}, \alpha^{\prime}=\alpha_{\mu}^{\prime}\right)$

$$
w_{\alpha \rightarrow \alpha^{\prime}}^{(s)}=\frac{1}{\hbar^{2}} \int_{-\infty}^{\infty} \mathrm{d} \tau \mathrm{e}^{-\mathrm{i} \omega_{\alpha \alpha^{\prime}}^{(s)} \tau}\left\langle\mathrm{e}^{-\mathrm{i} H_{B} \tau / \hbar} \Phi_{\alpha \alpha^{\prime}}^{(s)} \mathrm{e}^{\mathrm{i} H_{B} \tau / \hbar} \Phi_{\alpha^{\prime} \alpha}^{(s)}\right\rangle .
$$

Here, $H_{B}$ is the bath Hamiltonian and $\varepsilon_{s \alpha}-\varepsilon_{s \alpha^{\prime}}=\hbar \omega_{\alpha \alpha^{\prime}}^{(s)}$ denote the transition energies. The bracket $\langle\cdots\rangle$ reflects the thermal average with respect to the equilibrium state of the thermal bath.

The vibrational transition $\alpha \rightarrow \alpha^{\prime}$ described by the last term on the right-hand side of Eq. (A.2) are defined via the same transition probabilities (A.3) as it is the case for the inverse life-times $\tau_{s \alpha}^{-1}=\gamma_{s \alpha}$. The latter specify the relaxation time. For $t \gg \tau_{s \alpha}$ all off-diagonal matrix elements related to same electronic level $s$, vanish while the diagonal matrix elements, i.e. the populations $P_{s \alpha}(t)=\rho_{s \alpha s \alpha}(t)$, describe a thermal equilibrium (Boltzmann) distribution versus the states $\left|\chi_{s \alpha}\right\rangle$. Accordingly we may write

$$
\rho_{s \alpha s \beta}(t)=\delta_{\alpha, \beta} W\left(\varepsilon_{s \alpha}\right) P_{s}(t),
$$

where

$$
P_{s}(t)=\sum_{\alpha} P_{s \alpha}(t)
$$

denotes the integral population of the sth electronic level. Furthermore, we introduced the Boltzmann distribution of the vibrational states of the sth electronic level

$$
W\left(\varepsilon_{s \alpha}\right)=Z_{s}^{-1} \mathrm{e}^{-\left(\varepsilon_{s x}-\varepsilon_{s 0}\right) / k_{\mathrm{B}} T} \quad\left(Z_{s}=\sum_{\alpha} \mathrm{e}^{-\left(\varepsilon_{s x}-\varepsilon_{s 0}\right) / k_{\mathrm{B}} T}\right) .
$$

Relation (A.4) represents a coarse-grained description [73,81] describing the electron transmission via kinetic equations for integral populations $P_{s}(t)$ (in our case, for $P_{\mu \sigma_{\mu}}(t)$ ) only.

If one inserts the factorized distribution (A.4) into Eq. (A.2) and takes into consideration that $w_{\alpha \rightarrow \alpha^{\prime}}^{(s)} / w_{\alpha^{\prime} \rightarrow \alpha}^{(s)}=$ $\exp \left[\left(\varepsilon_{s \alpha}-\varepsilon_{s \alpha^{\prime}}\right) / k_{\mathrm{B}} T\right]$, the following set of equations for diagonal elements

$$
\dot{\rho}_{s \alpha . s \alpha}(t)=\frac{\mathrm{i}}{\hbar}\left[\rho(t), V_{\mathrm{int}}\right]_{s \alpha . s \alpha}
$$

as well as off-diagonal elements

$$
\dot{\rho}_{s \alpha s^{\prime} \beta}(t)=-\frac{\mathrm{i}}{\hbar} \Delta E_{s \alpha s^{\prime} \beta} \rho_{s \alpha s^{\prime} \beta}(t)+\frac{\mathrm{i}}{\hbar}\left[\rho(t), V_{\mathrm{int}}\right]_{s \alpha s^{\prime} \beta}
$$

is obtained. In the latter equation we introduced complex energy difference $\Delta E_{s \alpha s^{\prime} \beta}=E_{s \alpha}-E_{s^{\prime} \beta}^{*}$ with

$$
E_{s \alpha} \equiv \varepsilon_{s \alpha}-\mathrm{i} \gamma_{s \alpha} \text {. }
$$

These complex energies of the electron-vibration levels include level broadening $\gamma_{s \alpha}$ caused by an interaction with the heat bath. At $t \gg \tau_{s \alpha}$ the presence of this broadening allows to neglect the time derivative of $\rho_{s \alpha s^{\prime} \beta}(t)$, and a set of algebraic equations follows

$$
\rho_{s \alpha s^{\prime} \beta}(t)=\frac{1}{\Delta E_{s \alpha s^{\prime} \beta}}\left[\rho(t), V_{\mathrm{int}}\right]_{s \alpha s^{\prime} \beta} .
$$


Note that the description of a stationary electron transmission (related to a stationary current) requires $\dot{\rho}_{s \alpha s^{\prime} \beta}(t)=0$ and automatically transforms the set of Eqs. (A.8) to Eqs. (A.10).

Eqs. (A.7) and (A.10) can be represented in operator form if we introduce the projection operators $\hat{T}_{d}$ and $\hat{T}_{n d}=1-\hat{T}_{d}$. They should separate any operator $\hat{A}$ into diagonal and off-diagonal components. Noting $\rho_{d}(t)=\hat{T}_{d} \rho(t)$ and $\rho_{n d}(t)=\hat{T}_{n d} \rho(t)$, the following set of coupled operator equations is obtained:

$$
\begin{aligned}
& \dot{\rho}_{d}(t)=-\mathrm{i} \hat{T}_{d} \mathscr{L}_{V} \rho_{n d}(t), \\
& \rho_{n d}(t)=-\mathrm{i} \int_{0}^{\infty} \mathrm{d} \tau \mathscr{U}^{(0)}(\tau) \mathscr{L}_{V} \rho_{d}(t)-\mathrm{i} \int_{0}^{\infty} \mathrm{d} \tau \mathscr{U}^{(0)}(\tau) \mathscr{L}_{V} \rho_{n d}(t),
\end{aligned}
$$

with the time evolution superoperator defined as

$$
\mathscr{U}^{(0)}(\tau)=\mathrm{e}^{-\mathrm{i} \mathscr{Q}_{0} \tau} .
$$

In Eqs. (A.11) and (A.12), $\mathscr{L}_{0} \equiv(1 / \hbar)\left[H_{0}, \ldots\right]$ and $\mathscr{L}_{V} \equiv(1 / \hbar)\left[V_{\text {int }}, \ldots\right]$ denote the Liouville superoperators with respect to the molecular Hamiltonian

$$
H_{0}=\sum_{s \alpha} E_{s \alpha}|s \alpha\rangle\langle s \alpha|
$$

and with respect to the off-diagonal interaction $V_{\text {int }}$, respectively. Since $E_{s \alpha}$ is complex (see Eq. (A.9)) and thus $H_{0} \neq H_{0}^{+}$the time evolution superoperator $\mathscr{U}^{(0)}(\tau)$ acts on an arbitrary operator $A$ according to $\mathscr{U}^{(0)}(\tau) A=\exp \left(-\mathrm{i} H_{0} \tau / \hbar\right) A \exp \left(\mathrm{i} H_{0}^{+} \tau / \hbar\right)$. Next we note that the second equation of the set (A.11) couples the off-diagonal part of the density matrix to the diagonal one. Therefore, an iteration procedure can be introduced to get equations for $\rho_{d}(t)$ only. They read

$$
\dot{\rho}_{d}(t)=-2 \rho_{d}(t) \text {. }
$$

Here, 2 can be understood as a transmission superoperator which reads

$$
\mathscr{Q}=\sum_{k=1}^{\infty} \mathscr{Q}_{2 k} .
$$

Every term

$$
\mathscr{Q}_{2 k}=-(-\mathrm{i} / \hbar)^{2 k} \hat{T}_{d} \int_{0}^{\infty} \mathrm{d} \tau_{2 k-1} \int_{0}^{\infty} \mathrm{d} \tau_{2 k-2} \ldots \int_{0}^{\infty} \mathrm{d} \tau_{1} \mathscr{L}_{V} \hat{T}_{n d} \mathscr{U}^{(0)}\left(\tau_{2 k-1}\right) \mathscr{L}_{V} \hat{T}_{n d} \mathscr{U}^{(0)}\left(\tau_{2 k-2}\right) \ldots \hat{T}_{n d} \mathscr{U}^{(0)}\left(\tau_{1}\right) \mathscr{L}_{V}
$$

only contains an even number of operators $V_{\text {int }}$.

To derive kinetic equations for the integral populations $P_{s}(t)$ we rewrite the master equation (A.14) in matrix form

$$
\dot{\rho}_{s \alpha . s \alpha}(t)=-\sum_{s^{\prime} \beta}\left\langle s \alpha\left|\left(\mathscr{Q}\left|s^{\prime} \beta\right\rangle\left\langle s^{\prime} \beta\right|\right)\right| s \alpha\right\rangle \rho_{s^{\prime} \beta s^{\prime} \beta}(t) .
$$

An inspection of each term on the right-hand side of the kinetic equation (A.17) (by taking into account the expansion (A.15) and the relations (A.4) and (A.6)) shows that it can be transformed into the following balance equation:

$$
\dot{P}_{s}(t)=-\sum_{s^{\prime}}\left[\kappa_{s \rightarrow s^{\prime}} P_{s}(t)-\kappa_{s^{\prime} \rightarrow s} P_{s^{\prime}}(t)\right]
$$

The rate constants characterizing the $s \rightarrow s^{\prime}$ transition read

$$
\kappa_{s \rightarrow s^{\prime}}=\frac{2 \pi}{\hbar} \sum_{\alpha \beta}\left|T_{s \alpha, s^{\prime} \beta}\right|^{2} W\left(\varepsilon_{s \alpha}\right) L\left(\varepsilon_{s \alpha}-\varepsilon_{s^{\prime} \beta}\right) .
$$

The matrix elements $T_{s \alpha s^{\prime} \beta}=\left\langle s \alpha\left|V^{(\text {eff })}\right| s^{\prime} \beta\right\rangle$ which couple electron-vibrational states $s \alpha$ and $s^{\prime} \beta$ are defined via the effective transition operator

$$
V^{(\text {eff })}=V_{\text {int }}+V_{\text {int }} G(E) V_{\text {int }} .
$$

It contains the operator $V_{\text {int }}$ as well as Green's operator $G(E)=(E-H)^{-1}$, where $E=\varepsilon_{s \alpha} \approx \varepsilon_{s^{\prime} \beta}$ denotes the transition energy. The Hamiltonian $H=H_{0}+V_{\text {int }}$ contains two terms, where the first coincides with the effective molecular Hamiltonian (A.13) while the operator $V_{\text {int }}$ describes transitions between different electronic levels and is identical with $V_{M-M}$, Eq. (5). Note, that energies (A.9) of the Hamiltonian (A.13) include level broadenings $\gamma_{s \alpha}$ caused by vibrational relaxation. Moreover, 


$$
L\left(\varepsilon_{s \alpha}-\varepsilon_{s^{\prime} \beta}\right)=\frac{1}{\pi} \frac{\gamma_{s \alpha}+\gamma_{s^{\prime} \beta}}{\left(\varepsilon_{s \alpha}-\varepsilon_{s^{\prime} \beta}\right)^{2}+\left(\gamma_{s \alpha}+\gamma_{s^{\prime} \beta}\right)^{2}}
$$

denotes the normalized Lorentzian which reduces to a delta-function if $\gamma_{s \alpha}$ as well as $\gamma_{s^{\prime} \beta}$ vanish.

After a proper specification of the electron-vibrational states $|s \alpha\rangle$ Eqs. (A.18),(A.19),(A.20) can be used for a description of non-adiabatic as well as adiabatic transfer processes within the molecule. In the present consideration the extended states are used to characterize electronic properties of molecule. Therefore the derived kinetic equations (A.18) describe only relaxation transitions between extended electronic levels $s=\mu \sigma$ and $s^{\prime}=\mu^{\prime} \sigma$ caused by interaction $V_{\text {int }}=V_{M-M}$, Eq. (5).

\section{A.2. Coupling to the leads}

To account for the leads we have to introduce the lead electronic states $\left|L \mathbf{k} \sigma_{\mathbf{k}}\right\rangle$ and $\left|R \mathbf{q} \sigma_{\mathbf{q}}\right\rangle$ which complement the molecular electron-vibrational states $|s \alpha\rangle$. If the fast relaxation discussed beforehand is only present in the molecule we arrive at the generalized set of kinetic equations

$$
\dot{P}_{j}(t)=-\sum_{j^{\prime}}\left[K_{j \rightarrow j^{\prime}} P_{j}(t)-K_{j^{\prime} \rightarrow j} P_{j^{\prime}}(t)\right]
$$

where $j$ and $j^{\prime}$ refer to all LMR electronic states. If $j=s=\mu$ and $j^{\prime}=s^{\prime}=\mu^{\prime}$ the rate constants $K_{j \rightarrow j^{\prime}}$ coincide with those given by Eqs. (A.19) and (A.20). However, the transition operator (A.20) now contains Green's operator defined by the electron-vibrational Hamiltonian $H=H_{L M R}^{(\mathrm{e}-\mathrm{v})}$ of the total LMR system. $H_{L M R}^{(\mathrm{e}-\mathrm{v})}$ contains three parts. The first corresponds to the molecular Hamiltonian (4), however with energies $\varepsilon_{\mu \alpha_{\mu}}$ replaced by $E_{\mu \alpha_{\mu}}$, Eq. (A.9). The second part refers to the lead Hamiltonian, Eq. (7), and the third one coincides with the interaction between the leads and the molecule, Eq. (8). After introducing the abbreviations $r k \equiv r \mathbf{k} \lambda, \xi \equiv \mu \alpha_{\mu}$ the Hamiltonian reads (spin quantum numbers are timely omitted)

$$
H_{L M R}^{(\mathrm{e}-\mathrm{v})}=\sum_{r k} E_{r k}|r k\rangle\left\langle r k\left|+\sum_{\xi} E_{\xi}\right| \xi\right\rangle\langle\xi|+\sum_{r k, \xi}\left[V_{r k, \xi}|r k\rangle\langle\xi|+\text { h.c. }\right] .
$$

If $j$ indicates one of lead states (for instance, $j=L \mathbf{k}$ ) while $j^{\prime}=s=\mu$ refers to the $\mu$ th molecular level then

$$
K_{L \mathbf{k} \rightarrow \mu}=\frac{2 \pi}{\hbar} \sum_{\alpha_{\mu} \lambda}\left|T_{L \mathbf{k} \lambda, \mu \alpha_{\mu}}\right|^{2} f_{L}\left(E_{L \mathbf{k}}\right) W\left(\varepsilon_{\lambda}^{(0)}\right) L\left(E_{L \mathbf{k}}-\Delta \varepsilon_{\mu \alpha_{\mu}, \lambda}\right) .
$$

Here, the quantity

$$
\Delta \varepsilon_{\mu \alpha_{\mu}, \lambda}=\varepsilon_{\mu \alpha_{\mu}}-\varepsilon_{\lambda}^{(0)}
$$

is the difference between molecular electron-vibrational energies valid if an extra electron does or does not populates the molecule. The summation in Eq. (A.24) covers the vibrational states of the molecule valid if it does and does not contain an extra electron (indexes $\alpha_{\mu}$ and $\lambda$, respectively). The factor $f_{L}\left(E_{L \mathbf{k}}\right) W\left(\varepsilon_{\lambda}^{(0)}\right)$ gives the probability that the molecule is in the $\lambda$ th vibrational state before the extra electron enters. Note the introduction of the Fermi function of the left lead

$$
f_{L}\left(E_{L \mathbf{k}}\right)=\left\{\exp \left[\left(E_{L \mathbf{k}}-E_{\mathrm{F}}\right) / k_{\mathrm{B}} T\right]+1\right\}^{-1},
$$

where $E_{\mathrm{F}}$ is the Fermi energy of the lead and the introduction of

$$
W\left(\varepsilon_{\lambda}^{(0)}\right)=Z_{0}^{-1} \mathrm{e}^{-\left(\varepsilon_{\lambda}^{(0)}-\varepsilon_{0}^{(0)}\right) / k_{\mathrm{B}} T} \quad\left(Z_{0}=\sum_{\alpha} \mathrm{e}^{-\left(\varepsilon_{\lambda}^{(0)}-\varepsilon_{0}^{(0)}\right) / k_{\mathrm{B}} T}\right)
$$

as well as of the Lorentzian

$$
L\left(E_{L \mathbf{k}}-\Delta \varepsilon_{\mu \alpha_{\mu}, \lambda}\right)=\frac{1}{\pi} \frac{\gamma_{\lambda}+\gamma_{\mu \alpha_{\mu}}}{\left(E_{L \mathbf{k}}-\Delta \varepsilon_{\mu \alpha_{\mu}, \lambda}\right)^{2}+\left(\gamma_{\lambda}+\gamma_{\mu \alpha_{\mu}}\right)^{2}} .
$$

Lorentzian contains the broadening of the molecular electron-vibrational levels involved in the lead-molecule electron transition. The matrix element $T_{L \mathbf{k} \lambda, \mu \alpha_{\mu}}=\left\langle L \mathbf{k} \lambda\left|V^{(\mathrm{eff})}\right| \mu \alpha_{\mu}\right\rangle$ has to be calculated with the transition operator (A.20) where $E=E_{L \mathbf{k}}+\varepsilon_{\lambda}^{(0)} \approx \varepsilon_{\mu \alpha_{\mu}}$ and $H=H_{L M R}^{(\mathrm{e}-\mathrm{v})}$. 
If the electron transmission occurs between the leads then the left-to-right rate constant reads

$$
K_{L \mathbf{k} \rightarrow R \mathbf{q}}=\frac{2 \pi}{\hbar} \sum_{\lambda \lambda^{\prime}}\left|T_{L \mathbf{k} \lambda, R \mathbf{q} \lambda^{\prime}}\right|^{2} f_{L}\left(E_{L \mathbf{k}}\right)\left(1-f_{R}\left(E_{R \mathbf{q}}-e V\right)\right) W\left(\varepsilon_{\lambda}^{(0)}\right) L\left(E_{L \mathbf{k}}-E_{R \mathbf{q}}+\Delta \varepsilon_{\lambda, \lambda^{\prime}}^{(0)}\right)
$$

This formula is based on the supposition that the left lead is fixed at zero voltage $\left(V_{L}=0\right)$ so that a lead-lead voltage bias is defined by the potential $V=V_{R}$ applied to the right lead. The Lorentzian

$$
L\left(E_{L \mathbf{k}}-E_{R \mathbf{q}}+\Delta \varepsilon_{\lambda, \lambda^{\prime}}^{(0)}\right)=\frac{1}{\pi} \frac{\gamma_{\lambda}+\gamma_{\lambda^{\prime}}}{\left(E_{L \mathbf{k}}-E_{R \mathbf{q}}+\Delta \varepsilon_{\lambda, \lambda^{\prime}}^{(0)}\right)^{2}+\left(\gamma_{\lambda}+\gamma_{\lambda^{\prime}}\right)^{2}}
$$

takes into consideration the relaxation processes in the molecule when the latter does not contain an extra electron. Eq. (A.30) reflects the fact that during the lead-to-lead transmission the molecule can gain or lose the vibrational energy $\Delta \varepsilon_{\lambda, \lambda^{\prime}}^{(0)}=\varepsilon_{\lambda}^{(0)}-\varepsilon_{\lambda^{\prime}}^{(0)}$. The matrix elements $V_{L \mathbf{k} \lambda, R \mathbf{q} \lambda^{\prime}}=\left\langle L \mathbf{k} \lambda\left|V^{(\text {eff })}\right| R \mathbf{q} \lambda^{\prime}\right\rangle$ are calculated with the transition operator, Eq. (A.20), where $E=E_{L \mathbf{k}}+\varepsilon_{\lambda}^{(0)} \approx E_{R \mathbf{q}}+\varepsilon_{\lambda^{\prime}}^{(0)}$ and $H=H_{L M R}^{(\mathrm{e}-\mathrm{v})}$.

\section{A.3. The effective electron-vibrational Hamiltonian}

The transition matrix elements which enter the rate constants given in the preceding section have to be calculated in using the transition operator (A.20). It contains two contributions, the first, $V_{\mathrm{int}}=V_{M-M}+V_{L R-M}$, characterizes the direct coupling between molecular electron-vibrational states and between molecular levels and those of the leads (cf. $V_{M-M}$, Eq. (5) as well as $V_{L R-M}$, Eq. (8), respectively). Therefore, one can calculate the corresponding rate constants in using the following approximation for the matrix elements:

$$
T_{s \alpha, s^{\prime} \beta} \approx\left\langle\mu \alpha_{\mu}\left|V_{M-M}\right| \mu^{\prime} \beta_{\mu^{\prime}}\right\rangle=V_{\mu \alpha_{\mu}, \mu^{\prime} \beta_{\mu^{\prime}}}
$$

and

$$
T_{L \mathbf{k} \lambda, \mu \alpha_{\mu}} \approx\left\langle L \mathbf{k} \lambda\left|V_{L R-M}\right| \mu \alpha_{\mu}\right\rangle=V_{L \mathbf{k} \lambda, \mu \alpha_{\mu}} .
$$

These expressions demonstrate that in the framework of the chosen approximation the noted matrix elements are expressed through those specifying the interactions (5) and (8), respectively.

The second part of $V^{(\mathrm{eff})}$ is given by $V_{\mathrm{int}} G(E) V_{\mathrm{int}}$ which may account for complex dynamic processes within LMR system, in particular coupling the electronic states of the left and the right lead

$$
T_{L \mathbf{k} \lambda, R \mathbf{q} \lambda^{\prime}}=\left\langle L \mathbf{k} \lambda\left|V_{\text {int }} G(E) V_{\text {int }}\right| R \mathbf{q} \lambda^{\prime}\right\rangle
$$

To make the expression more concrete we note that Green's operator is defined via the electron-vibrational Hamiltonian $H=H_{L M R}^{(\mathrm{e}-\mathrm{v})}$, Eq. (A.23). Let us suppose that $|F\rangle$ and $\mathscr{E}_{F}$ are the eigenstates and eigenvalues of the $H_{L M R}^{(\mathrm{e}-\mathrm{v})}$. In this case Green's operator reduces to

$$
G(E)=\sum_{F} \frac{|F\rangle\langle F|}{E-\mathscr{E}_{F}}
$$

and thus we get

$$
T_{L \mathbf{k} \lambda, R \mathbf{q} \lambda^{\prime}}=\sum_{F} \frac{V_{L \mathbf{k} \lambda, F} V_{F, \lambda^{\prime} R \mathbf{q}}}{E-\mathscr{E}_{F}}
$$

Here, $V_{L \mathbf{k} \lambda, F}=\left\langle L \mathbf{k} \lambda\left|V_{L R-M}\right| F\right\rangle$ and $V_{F, \lambda^{\prime} R \mathbf{q}}=\left\langle F\left|V_{L R-M}\right| \lambda^{\prime} R \mathbf{q}\right\rangle$ are the matrix elements between the electronic lead states $|\lambda L \mathbf{k}\rangle$ and $\left|\lambda^{\prime} R \mathbf{q}\right\rangle$ and the LMR states $|F\rangle$. To obtain the energies $\mathscr{E}_{F}$ and the states $|F\rangle$ we have to diagonalize the Hamiltonian (A.23) using the transformation

$$
|F\rangle=\sum_{r k} \Theta_{F}(r k)|r k\rangle+\sum_{\xi} \Theta_{F}(\xi)|\xi\rangle .
$$

The $\Theta_{F}(r k)$ and $\Theta_{F}(\xi)$ as well as the eigenvalues $\mathscr{E}_{F}$ are derived from the set of equations

$$
\left(E_{r k}-\mathscr{E}_{F}\right) \Theta_{F}(r k)+\sum_{\xi} V_{r k, \xi}^{*} \Theta_{F}(\xi)=0, \quad\left(E_{\xi}-\mathscr{E}_{F}\right) \Theta_{F}(\xi)+\sum_{r=L, R} \sum_{k} V_{r k, \xi} \Theta_{F}(r k)=0 .
$$

It follows from the first equation of this set that

$$
\Theta_{F}(r k)=\frac{1}{\mathscr{E}_{F}-E_{r k}} \sum_{\xi} V_{r k, \xi}^{*} \Theta_{F}(\xi) .
$$


Substituting this relation into the second equation (A.37) leads to the reduced set of equations

$$
\sum_{\xi^{\prime}}\left[\left(E_{\xi}-E\right) \delta_{\xi \xi^{\prime}}+\Sigma_{\xi \xi^{\prime}}^{(L)}(E)+\Sigma_{\xi \xi^{\prime}}^{(R)}(E)\right] \Theta_{F}\left(\xi^{\prime}\right)=0
$$

where we have introduced the self-energies

$$
\Sigma_{\xi^{\prime} \xi^{\prime}}^{(r)}(E)=\sum_{k} \frac{V_{r k, \xi}^{*} V_{r k, \xi^{\prime}}}{E-E_{r k}+\mathrm{i} 0^{+}}
$$

The set is extremely appropriated to derive approximate expression for the energies $E=\mathscr{E}_{F}$ as well as for the coefficients $\Theta_{F}(r k)$ and $\Theta_{F}(\xi)$. Below we restrict ourself to the case where the leads are considered as a macroscopic system. Therefore, both leads perturb the molecular states in a much stronger way then the molecule perturbed the leads. This fact allows to split off the extended LMR states $|F\rangle$ into two types [79]. The first type is identical to the lead states $|r k\rangle$. It means that

$$
\Theta_{F(r k)}(\xi) \approx 0
$$

and thus $|F\rangle=|F(r k)\rangle \approx|r k\rangle$. The corresponding eigenvalues $\mathscr{E}_{F}$ coincide with the unperturbed energies $E_{r \mathbf{k}}(r=L, R)$ of the leads, i.e. $\mathscr{E}_{F}=\mathscr{E}_{F(r k)} \approx E_{r \mathbf{k}}$. The second type of LMR states coincides with molecular electron-vibrational states, $|F\rangle=|F(\xi)\rangle \approx \sum_{\xi} \Theta_{F}(\xi)|\xi\rangle$. Due to the condition (A.41) the set (A.39) is reduced to the subset

$$
\sum_{\xi^{\prime}}\left[\left\langle\xi\left|H_{M}^{(\mathrm{eff})}\right| \xi^{\prime}\right\rangle-E \delta_{\xi \xi^{\prime}}\right] \Theta_{f \alpha_{f}}\left(\xi^{\prime}\right)=0
$$

where the notation $f \alpha_{f} \equiv F(\xi)$ for the molecular electron-vibrational states formed from initial molecular electronvibrational states $|\xi\rangle=\left|\mu \alpha_{\mu}\right\rangle$ is employed. Here, we had introduced the effective molecular Hamiltonian

$$
H_{M}^{(\text {eff })}=\sum_{\xi} E_{\xi}|\xi\rangle\langle\xi|+\hat{\Sigma}^{(L)}+\hat{\Sigma}^{(R)}
$$

which apart from the main contribution (the first term on the right-hand side) contains self-energy operators defined by Eq. (A.40). The solution of Eq. (A.42) is essentially simplified by the fact that self-energies exhibit a negligible dependence on the energy $E[10,55,60,61]$. Therefore, the diagonalization of $H_{M}^{\text {(eff) }}$ represents an algebraic problem only. Denoting the eigenenergies of this Hamiltonian by $\mathscr{E}_{f \alpha_{f}}$ one obtains the form (17). In the case of macroscopic leads the influence of the molecular states on the lead states may be ignored. According to relation (A.38) and condition (A.41), we obtain

$$
\Theta_{F(r k)}\left(r^{\prime} k^{\prime}\right) \approx \delta_{r, r^{\prime}} \delta_{k, k^{\prime}}
$$

and may conclude that only those matrix elements $\left\langle L \mathbf{k} \lambda\left|V_{L R-M}\right| F\right\rangle$ do not vanish where $F=F(\xi) \equiv f \alpha_{f}$. Noting this fact the matrix elements (A.35) reduce to

$$
V_{L \mathbf{k} \lambda, R \mathbf{q} \lambda^{\prime}}=\sum_{\xi} \sum_{\xi^{\prime}} V_{L \mathbf{k} \lambda, \xi} G_{\xi^{\prime} \xi^{\prime}} V_{R \mathbf{q} \lambda^{\prime}, \xi^{\prime}}^{*} \cdot
$$

Here, the couplings $V_{r \mathbf{k} \lambda, \xi} \equiv V_{r \mathbf{k} \lambda, \mu \alpha_{\mu}}(r=L, R)$ are defined by Eq. (9), while the matrix elements of Green's operator read

$$
G_{\xi \xi^{\prime}}=\sum_{f \alpha_{f}} \frac{\Theta_{f \alpha_{f}}(\xi) \Theta_{f \alpha_{f}}^{*}\left(\xi^{\prime}\right)}{E-\mathscr{E}_{f \alpha_{f}}} .
$$

This expression shows that only in the case of macroscopic electrodes the calculation of the LMR Green's operator can be replaced by the calculation of molecular Green's operators defined via the effective Hamiltonian (A.43). The latter includes imaginary parts caused by the interaction of the molecule with a heat bath as well as with the lead. Obviously, these interactions result in broadening of the molecular electron-vibrational levels.

\section{References}

[1] F.L. Carter (Ed.), Molecular Electronic Devices, Marcel Dekker, New York, 1982.

[2] A.J. Aviram, Am. Chem. Soc. 110 (1988) 5687.

[3] M. Kemp, V. Mujica, M.A. Ratner, J. Chem. Phys. 101 (1994) 5172.

[4] V. Mujica, M. Kemp, M.A. Ratner, J. Chem. Phys. 101 (1994) 6849. 
[5] V. Mujica, M. Kemp, A. Roitberg, M.A. Ratner, J. Chem. Phys. 104 (1996) 7296.

[6] E.G. Petrov, I.S. Tolokh, A.A. Demidenko, V.V. Gorbach, Chem. Phys. 193 (1995) 237.

[7] M. Ratner, J. Jortner (Eds.), Molecular Electronics, Blackwell Science, Oxford, 1997.

[8] A. Mujica, A. Nitzan, Y. Mao, W. Davis, M. Kemp, A. Roitberg, M.A. Ratner, in: J. Jortner, M. Bixon (Eds.), Electron Transfer - from Isolated Molecules to Biomolecules, Advances in Chemical Physics Series, vol. 107, John Wiley, New York, 1999, p. 403.

[9] V. May, J. Mol. Electron. 6 (1990) 187; V. May, Phys. Lett. A 161 (1991) 118.

[10] S.N. Yaliraki, M. Kemp, M. Ratner, J. Am. Chem. Soc. 121 (1999) 3428.

[11] P. Hänggi, M. Ratner, S. Yaliraki, Chem. Phys. 281 (2002) 111.

[12] E.G. Petrov, I.S. Tolokh, V. May, Phys. Rev. Lett. 79 (1997) 4006.

[13] I.A. Goychuk, E.G. Petrov, V. May, Phys. Rev. E. 56 (1997) 1421.

[14] I.A. Goychuk, E.G. Petrov, V. May, J. Chem. Phys. 106 (1997) 4522.

[15] J. Lehmann, S. Kohler, V. May, P. Hänggi, J. Chem. Phys. 121 (2004) 2278.

[16] S. Camalet, J. Lehmann, S. Kohler, P. Hänggi, Phys. Rev. Lett. 90 (2003) 210602;

S. Camalet, S. Kohler, P. Hänggi, Phys. Rev. B 70 (2004) 155326;

S. Kohler, J. Lehmann, P. Hänggi, Driven quantum transport on the nanoscale, Phys. Rep. (2005). cond-mat/0409251.

[17] D.N. Beratan, J.N. Betts, J.N. Onuchic, Science 252 (1991) 1285.

[18] J.W. Evenson, M. Karplus, J. Chem. Phys. 96 (1992) 5272.

[19] W.B. Davis, M.R. Wasielewski, M.A. Ratner, V. Mujica, A. Nitzan, J. Phys. Chem. 101 (1997) 6158.

[20] M. Grifoni, P. Hänggi, Phys. Rep. 304 (1998) 229.

[21] E.G. Petrov, Ukr. J. Phys. 43 (1998) 1630.

[22] D. Segal, A. Nitzan, W.B. Davis, M.R. Wasilewski, M. Ratner, J. Phys. Chem. B 104 (2000) 3817.

[23] E.G. Petrov, I.S. Tolokh, V. May, J. Chem. Phys. 109 (1998) 9561.

[24] A. Nitzan, J. Phys. Chem. A 105 (2001) 2677.

[25] A.M. Kuznetsov, J. Ulstrup, J. Chem. Phys. 116 (2002) 2149.

[26] E.G. Petrov, V. May, P. Hänggi, Chem. Phys. 296 (2004) 251.

[27] N.B. Zhitinev, H. Meng, Z. Bao, Phys. Rev. Lett. 88 (2002) 226801.

[28] S. Tikhodeev, M. Natario, K. Makoshi, T. Mii, H. Ueba, Surf. Sci. 493 (2001) 63.

[29] T. Mii, S.G. Tikhodeev, H. Ueba, Phys. Rev. B 68 (2003) 205406.

[30] X.H. Qui, G.V. Nazin, W. Ho, Phys. Rev. Lett. 92 (2004) 206102.

[31] M. Galperin, M.A. Ratner, A. Nitzan, J. Chem. Phys. 121 (2004) 11965.

[32] M.H. Hettler, H. Schoeller, W. Wentzel, Europhys. Lett. 57 (2002) 571.

[33] V. Mujica, A. Nitzan, S. Datta, M.A. Ratner, C.P. Kubiak, J. Phys. Chem. B 107 (2003) 91.

[34] A. Orella, F. Claro, Phys. Rev. Lett. 90 (2003) 178302.

[35] S.K. Pati, J. Phys. Chem. 118 (2003) 6529.

[36] S. Datta, Nanotechnology 15 (2004) S433.

[37] J.L. Bredas, R. Silbey (Eds.), Conjugated Polymers, Kluwer, Dordrecht, 1991.

[38] W.R. Salaneck, I. Lundstrom, B. Ranby, Conjugated Polymers and Related Compounds, Oxford University Press, Oxford, 1993.

[39] J.-M. Lehn, Supramolecular Chemistry, VCH Publisher, Weinheim, 1995.

[40] T.J. Marks, Science 227 (1985) 881.

[41] M.C. Petty, M.R. Brice, D. Bloor (Eds.), Introduction to Molecular Electronics, Oxford University Press, Oxford, 1995.

[42] D. Mihailovic, Europhys. News 28 (1997) 78.

[43] D. Gatteschi, Adv. Mater. 6 (1994) 635.

[44] O. Kahn, Molecular Magnetism, VCH Publishers, New York, 1993.

[45] H.B. Weber, J. Reichert, F. Weigend, R. Ochs, D. Beckmann, M. Mayor, R. Ahlrichs, H. von Löhneysen, Chem. Phys. 281 (2002) 113.

[46] J. Chen, M.A. Reed, Chem. Phys. 281 (2002) 127.

[47] C. Kergueris, J.-P. Bourgoin, S. Palacin, D. Esteve, C. Urbana, M. Magoga, C. Joachim, Phys. Rev. B 59 (1999) 12505.

[48] X.D. Cui, A. Primak, X. Zarate, J. Tomfohr, O.F. Sankey, A.L. Moore, T.A. Moore, D. Gust, G. Harris, S.M. Lindsay, Science 294 (2001) 571.

[49] M. Toerker, T. Fritz, H. Proehl, Phys. Rev. B 65 (2002) 245422.

[50] D.J. Wold, R. Haag, M.A. Rampi, C.D. Frisbie, J. Phys. Chem. 106 (2002) 2813.

[51] B. Xu, N.J. Tao, Science 301 (2003) 1221.

[52] K. Stokbro, J. Taylor, M. Brandbyge, J. Am. Chem. Soc. 125 (2003) 3674.

[53] F. Jäckel, Z. Wang, M.D. Watson, K. Müllen, J.P. Rabe, Chem. Phys. Lett. 387 (2004) 372.

[54] M.A. Reed, C. Zhou, C.J. Miller, T.P. Burgin, J.M. Tour, Science 278 (1997) 252.

[55] W. Tian, S. Datta, S. Hong, R. Reifenberger, J.I. Henderson, C.I. Kubiak, J. Chem. Phys. 109 (1998) 2874.

[56] P.A. Derosa, J.M. Seminario, J. Phys. Chem. B 105 (2001) 471.

[57] P. Damle, A.W. Ghosh, S. Datta, Chem. Phys. 281 (2002) 171.

[58] R. Landauer, Philos. Mag. 21 (1970) 863;

R. Landauer, Phys. Lett. A 85 (1981) 91.

[59] M. Büttiker, Phys. Rev. B 33 (1986) 3020.

[60] S. Datta, Electronic Transport in Mesoscopic Systems, Cambridge University Press, New York, 1995.

[61] A. Nitzan, Annu. Rev. Phys. Chem. 52 (2001) 681.

[62] A. Nitzan, M.A. Ratner, Science 300 (2003) 1384.

[63] E.G. Petrov, P. Hänggi, Phys. Rev. Lett. 86 (2001) 2862.

[64] E.G. Petrov, V. May, P. Hänggi, Chem. Phys. 281 (2002) 211.

[65] D. Segal, A. Nitzan, Chem. Phys. 268 (2001) 315;

D. Segal, A. Nitzan, Chem. Phys. 281 (2002) 235. 
[66] B. Fain, Theory of Rate Processes in Condensed Media, Springer, Berlin, 1980.

[67] S. Mukamel, Principles of Nonlinear Optical Spectroscopy, Oxford University, New York, 1995.

[68] K. Blum, Density Matrix Theory and Application, second ed., Plenum Press, New York, 1996.

[69] V. May, O. Kühn, Charge and Energy Transfer Dynamics in Molecular Systems, Wiley-VCH, Berlin, 2004.

[70] E.G. Petrov, Ye.V. Shevchenko, V. May, Chem. Phys. 288 (2003) 269.

[71] E.G. Petrov, Ya.R. Zelinskyy, V. May, J. Phys. Chem. B 108 (2004) 13208.

[72] F.K. Fong, Theory of Molecular Relaxation, John Wiley, New York, 1975.

[73] E.G. Petrov, Ya.R. Zelinskyy, V. May, J. Phys. Chem. B 106 (2002) 3092.

[74] J.T. Taylor, M. Brandbyge, R. Stokbro, Phys. Rev. Lett. 89 (2002) 138301.

[75] H. Bash, M.A. Ratner, J. Chem. Phys. 120 (2004) 5771.

[76] A. Marchenko, N. Katsonis, D. Fichou, C. Aubert, M. Malarcia, J. Am. Chem. Soc. 124 (2002) 9998.

[77] A.K. Felts, W.T. Pollard, R.A. Friesner, J. Chem. Phys. 99 (1995) 2929.

[78] J. Hubbard, Proc. R. Soc. London A 276 (1963) 238.

[79] E.G. Petrov, Low Temp. Phys. 31 (2005).

[80] If the Coulomb contribution to the gap (54) strongly exceeds the single-electron unbiased gap $\Delta E_{L}(0)\left(\Delta E_{R}(0)\right)$ specific transmission conditions through the already occupied separate energy level $\mu=1$ follow. The transmission through this occupied energy level becomes only possible, if $|e V|$ exceeds $U \Phi(11 ; 11) / \eta \sim U$. This means that the lowest and well separated extended molecular level begins to imitate the "metallic" properties of the molecule.

[81] E.G. Petrov, Y.e.V. Shevchenko, V.I. Teslenko, V. May, J. Chem. Phys. 115 (2001) 7107;

E.G. Petrov, V. May, J. Phys. Chem. A 105 (2001) 10176.

[82] C. Krzeminski, C. Delerue, G. Allan, Vuillaume, Phys. Rev. B 64 (2001) 085405.

[83] F. Zahid, M. Paulsson, S. Datta, in: Morkos (Ed.), Advanced Semiconductors and Organic Nano-techniques, Academic Press, New York, 2003, p. 41 (Chapter 2). 